\title{
Quantitative investigations of quantum coherence for a light-harvesting protein at conditions simulating photosynthesis
}

\author{
Daniel B. Turner, ${ }^{a}$ Rayomond Dinshaw, ${ }^{a}$ Kyung-Koo Lee, ${ }^{a}$ Michael S. Belsley, ${ }^{b}$ \\ Krystyna E. Wilk, ${ }^{c}$ Paul M. G. Curmi ${ }^{c}$ and Gregory D. Scholes ${ }^{* a}$
}

Received 21st November 2011, Accepted 27th January 2012

DOI: $10.1039 / \mathrm{c} 2 \mathrm{cp} 23670 \mathrm{~b}$

\begin{abstract}
Recent measurements using two-dimensional electronic spectroscopy (2D ES) have shown that the initial dynamic response of photosynthetic proteins can involve quantum coherence. We show how electronic coherence can be differentiated from vibrational coherence in 2D ES. On that basis we conclude that both electronic and vibrational coherences are observed in the phycobiliprotein light-harvesting complex PC645 from Chroomonas sp. CCMP270 at ambient temperature. These light-harvesting antenna proteins of the cryptophyte algae are suspended in the lumen, where the $\mathrm{pH}$ drops significantly under sustained illumination by sunlight. Here we measured 2D ES of PC645 at increasing levels of acidity to determine if the change in $\mathrm{pH}$ affects the quantum coherence; quantitative analysis reveals that the dynamics are insensitive to the $\mathrm{pH}$ change.
\end{abstract}

\section{Introduction}

Light-harvesting proteins - the primary receptors of sunlight in plants, algae, and bacteria - capture photons and then transfer the resulting excitation energy to a reaction centre, which is a component of a larger complex called a photosystem (PS). ${ }^{1,2}$ In this work we thoroughly examine experiments that reveal evidence for quantum coherence in light-harvesting dynamics. We focus on two questions. First is the quantum coherence and therefore the light-harvesting mechanism affected by the change in physiological conditions associated with photosynthetic activity? In particular, how robust are cryptophyte light-harvesting complexes to $\mathrm{pH}$ changes on the lumenal side of the thylakoid membrane? Second, we demonstrate how to discriminate between electronic and vibrational coherences and show that both are present in signals from the lightharvesting complex we have studied.

Recently, the femtosecond spectroscopy of photosynthetic light-harvesting proteins has been investigated intensely. Initially assumed to involve only incoherent relaxation dynamics, mounting experimental evidence suggests that the response involves a coherent oscillatory component. ${ }^{3-11}$ Some have used the experimental results to test new theories of energy transfer ${ }^{12-17}$ and to consider possible roles of quantum effects. ${ }^{18-21}$ Most of

${ }^{a}$ Department of Chemistry, Institute for Optical Sciences, and Centre for Quantum Information and Quantum Control, 80 St. George Street, Toronto, Ontario, M5S 3H6, Canada. E-mail: gscholes@chem.utoronto.ca

${ }^{b}$ Department of Physics, Center of Physics, University of Minho, Campus de Gualtar, 4710 - 057 Braga, Portugal

${ }^{c}$ School of Physics and Centre for Applied Medical Research,

St. Vincent's Hospital, The University of New South Wales, Sydney, New South Wales 2052, Australia the experimental studies used a technique known as twodimensional electronic spectroscopy (2D ES) - the optical analog of $2 \mathrm{D} \mathrm{NMR}^{22,23}$ - to probe dynamics using a sequence of femtosecond optical pulses to induce and then measure a third-order nonlinear optical response of the proteins. 2D ES enhances spectral selectivity beyond that achieved by many other femtosecond optical techniques such as pump-probe or transient-grating by spreading the spectral content over two frequency axes and then measuring dynamics by varying a third time period parametrically. ${ }^{24,25}$ Given that 2D ES was only recently developed, ${ }^{26,27}$ many research groups ${ }^{10,11,28-34}$ continue to develop experimental apparatus suitable for recording high-quality spectra. Studies have also pointed out the similarities ${ }^{35-42}$ and differences ${ }^{10}$ between the signatures of vibrational and electronic coherences in 2D ES. Below we isolate several contributions to the coherent dynamics by creation and manipulation of the 3D spectral solid (3D ES), which allows us to investigate the dynamics in detail.

Unlike in most photosynthetic organisms, in cryptophyte algae the increased acidification of the thylakoid lumen directly affects the local environment of the peripheral light-harvesting proteins. Here we performed 2D ES measurements of the lightharvesting protein phycocyanin 645 (PC645), isolated from the cryptophyte algae Chroomonas sp. CCMP270, at three $\mathrm{pH}$ levels. Through quantitative analysis, we expatiate on the contributions from vibrational and electronic coherences to the initial dynamics.

The splitting of water on the lumenal side of the thylakoid membrane by $\mathrm{PSII}^{43}$ —along with processes carried out by other protein complexes such as PSI, mobile electron carriers, and the cytochrome bf complex - results in the release of gaseous molecular oxygen, the reduction of nicotinamide-adenine 
dinucleotide phosphate $\left(\mathrm{NADP}^{+}\right)$to NADPH, and the generation of protons. The reduction of $\mathrm{NADP}^{+}$in the stroma and the release of protons in the lumen establish a $\mathrm{pH}$ gradient across the thylakoid membrane. In a drive toward equilibrium, the excess protons in the lumen power the membrane-bound ATP-synthase protein, which converts adenosine diphosphate (ADP) to the primary cellular energy carrier, adenosine triphosphate (ATP).

Under intense illumination, the reaction centres of photosynthetic organisms struggle to keep pace with the large influx of excitation energy. Organisms are capable of redirecting the excess excitation energy safely. The redirection mechanism is regulated by the $\mathrm{pH}$ change produced during photosynthesis; the change in thylakoid lumen $\mathrm{pH}$ triggers a biochemical feedback process, described below, in which excess energy is dissipated as heat.

The ability to regulate light-harvesting processes is necessary for photosynthetic organisms to work efficiently under varying light conditions as the intensity and spectral content of sunlight can fluctuate significantly during the day. Light-harvesting antennae have evolved photoprotection responses, such as downregulation, to avoid damage in the case of excessive exposure to sunlight. ${ }^{44}$ Under conditions of excess sunlight a process known as non-photochemical quenching (NPQ) dissipates this excess energy as heat. ${ }^{45-47} \mathrm{NPQ}$ refers to all fluorescence quenching that is not directly related to charge separation and is composed of a de-excitation feedback mechanism known as energy-dependent quenching (qE), a slow photoinhibitory quenching mechanism, and state-transition quenching. ${ }^{48} \mathrm{qE}-$ which involves the protonation of specific PSII proteins ${ }^{45,46,48,49}$ - serves as the main component of NPQ and is the only mechanism that can respond rapidly (in seconds to minutes) to changes in light intensity. ${ }^{50}$

The mechanism by which fluorescence is quenched and excess energy is dissipated has been studied for many years. ${ }^{50}$ It has been suggested that the PsbS protein, a subunit of PSII, is indispensable for effective $\mathrm{qE}^{48,49}$ Low thylakoid lumen $\mathrm{pH}$ results in the protonation of two lumen-exposed glutamate residues on PsbS and induces zeaxanthin synthesis from violaxanthin. It was proposed that zeaxanthin binds to PsbS resulting in de-excitation of the chlorophyll molecules. ${ }^{48,49}$ However, in a recent report, qE-type quenching was observed in systems lacking the PsbS protein and that instead of being indispensable, the Psbs protein acts as a catalyst for $\mathrm{qE}$ formation. ${ }^{45}$ Regardless of the exact mechanism through which $\mathrm{qE}$ occurs, in the absence of $\mathrm{qE}$, excess sunlight can lead to an increased production of damaging reactive oxygen species as byproducts of photosynthesis. This is due to the increased probability that singlet chlorophyll will form triplet chlorophyll, which reacts with ground-state oxygen to form highly reactive oxygen species. ${ }^{48} \mathrm{qE}$ prevents reactive oxygen species from forming by providing an alternative nonradiative deactivation channel for singlet chlorophyll. In this manner, $\mathrm{qE}$ prevents photooxidative oxygen from causing pigment bleaching or cell death.

In most photosynthetic organisms, the light-harvesting machinery and the reaction centres are located in the thylakoid membrane. Some organisms also contain additional lightharvesting components such as chlorosomes or phycobilisomes attached to the stromal side of the thylakoid membrane. In all of
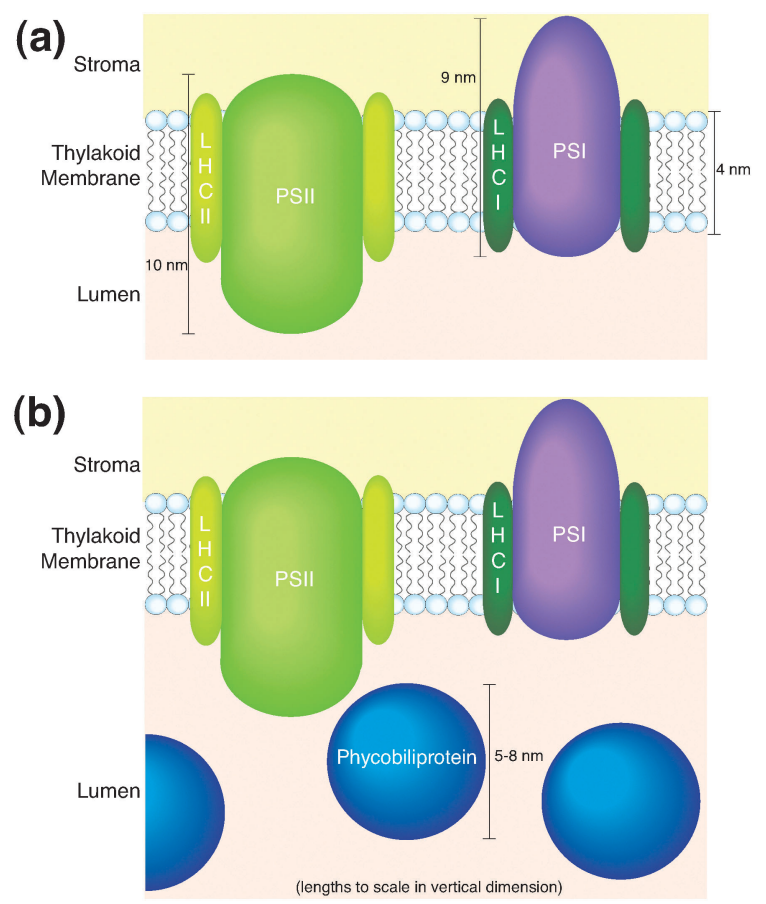

Fig. 1 The light-harvesting machinery of (a) most oxygenic photosynthetic organisms and of (b) cryptophyte algae. In plants and green algae, the plentiful membrane-bound LHC II are the primary lightabsorbing pigments. In cryptophytes, the primary light-absorbing pigments called phycobiliproteins are suspended in the aqueous environment of the thylakoid lumen. Only cryptophytes have antenna proteins located in the lumen.

these organisms, the light-harvesting machinery is not in the environment which changes $\mathrm{pH}$. In cryptophyte algae, however, the primary antenna proteins known as phycobiliproteins are found in the lumen. ${ }^{51}$ Phycobiliproteins transfer the absorbed excitation energy to chlorophyll- $a$ molecules located in the membrane-bound antenna complexes (LHC I and LHC II), which subsequently transfer the energy to the photosystems. ${ }^{43,52,53}$ The locale difference, depicted in Fig. 1, means that the lightharvesting antenna proteins of cryptophytes are bathed in an aqueous environment that is subject to a change in the $\mathrm{pH}$ level. Here we investigate the possibility that the $\mathrm{pH}$ drop alters the quantum-coherent dynamics in addition to triggering the quenching mechanism.

\section{Experimental}

A concentrated sample of purified PC645 isolated from Chroomonas sp. CCMP270 was frozen at $-20{ }^{\circ} \mathrm{C}$ until required for spectroscopic measurements. We prepared three $100 \mathrm{mM}$ sodium phosphate buffer solutions at varying $\mathrm{pH}$ levels by adjusting the relative amounts of $\mathrm{Na}_{2} \mathrm{HPO}_{4}$ and $\mathrm{NaH}_{2} \mathrm{PO}_{4}$; we measured the $\mathrm{pH}$ levels to be $5.7 \pm 0.1,6.5 \pm 0.1$, and $7.4 \pm 0.1$ using a calibrated microelectrode (Mettler Toledo). We diluted $100 \mu \mathrm{L}$ aliquots of concentrated protein into the three $1.5 \mathrm{~mL}$ volume buffer solutions; we measured and dispensed the solutions of protein and buffer using a micropipette to make the concentrations as equal as possible. The dilution produced samples with optical densities appropriate for $2 \mathrm{D}$ ES experiments, ${ }^{54}$ $\mathrm{OD}_{\lambda=645 \mathrm{~nm}} \approx 0.2$ in a $1 \mathrm{~mm}$ path-length cell. 
(a)

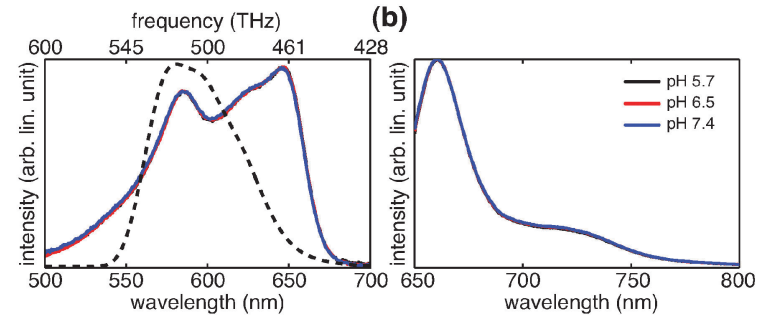

(c)

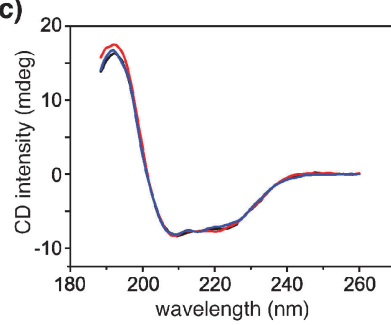

(e)

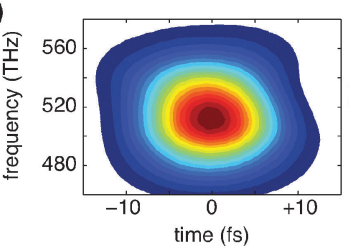

(d)

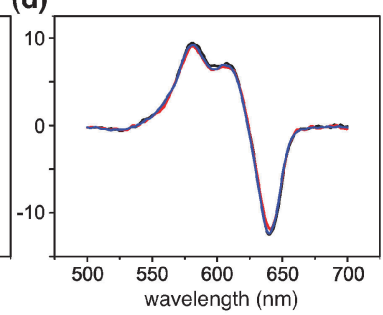

(f)

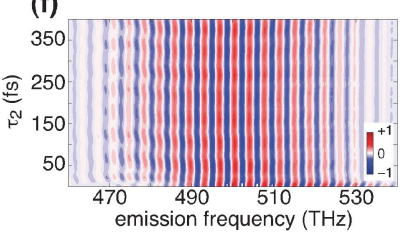

Fig. 2 Spectral characterization of PC645. The pH change had almost no impact on the linear spectra. (a) Absorption spectra (solid lines) and spectrum of the laser pulse (dashed line) used in the 2D ES experiments. (b) The three fluorescence spectra overlap almost perfectly. (c) UV and (d) visible circular-dichroism spectra. Traces in all four panels were corrected for small relative changes in concentration as described in the text. (e) Frequency-resolved optical gating (FROG) surface characterizing the pulse. (f) Wedge-calibration measurement described in the text

We recorded linear absorption spectra for each sample using a Varian Cary 100 Bio UV-visible absorption spectrophotometer which had a frequency resolution of $1 \mathrm{~nm}$. All spectra show a main peak centred at $645 \mathrm{~nm}$, a blue-shifted shoulder at $632 \mathrm{~nm}$, and a second distinct, but less intense, peak at $585 \mathrm{~nm}$. These spectral features are identical for all $\mathrm{pH}$ levels as shown in Fig. 2(a), where the three curves overlap almost perfectly. We normalized the spectra in Fig. 2(a) after recording the measured value of the optical density (at $645 \mathrm{~nm}$ ) to use as a normalization factor (for sample concentration) in the fluorescence and circular-dichroism (CD) measurements.

The normalized fluorescence spectra measured using a Varian Eclipse fluorescence spectrometer operated in the right-angle geometry are displayed in Fig. 2(b). Excitation occurred at $630 \mathrm{~nm}$; emission was monitored from $640-800 \mathrm{~nm}$; the excitation beam slit width was $5 \mathrm{~nm}$; and the emission slit width was $2.5 \mathrm{~nm}$. Each spectrum has a narrow peak $(\mathrm{FWHM} \approx 30 \mathrm{~nm})$ with a maximum at $661 \mathrm{~nm}$ and a vibronic sub-band in the vicinity of $720 \mathrm{~nm}$. The emission spectra of the three samples have identical lineshapes; however, the sample at $\mathrm{pH} 6.5$ was more stable than the samples at $\mathrm{pH} 5.7$ and 7.4, which rapidly decrease in intensity. After a 45 min wait time (no illumination), the fluorescence of the $\mathrm{pH} 6.5$ sample did not change while the intensity of the two other samples decreased by about $10 \%$.

We recorded CD spectra using a Jasco J-810 CD spectrometer equipped with a Jasco PTC-423S/15 temperature controller. The CD spectra in the near-UV wavelength range from 185 to $260 \mathrm{~nm}$ and in the visible range from 500 to $700 \mathrm{~nm}$ are shown in Fig. 2(c) and (d), respectively. The quartz sample cell had a pathlength of $1.0 \mathrm{~mm}$; the maximum sensitivity of the $\mathrm{CD}$ spectrometer was $100 \mathrm{mdeg}$; the spectral resolution was $0.5 \mathrm{~nm}$; the scanning speed was $100 \mathrm{~nm} \mathrm{~min}{ }^{-1}$ with a $2 \mathrm{~s}$ response time; and all presented spectra are averages of ten scans measured at $20.0 \pm 0.2{ }^{\circ} \mathrm{C}$. The $\mathrm{CD}$ spectra in this temperature range are relatively insensitive to temperature changes until about $45{ }^{\circ} \mathrm{C}$, above which we observed the proteins denature ${ }^{55}$ (data not shown). This test of denaturing was the only measurement not performed at room temperature (297 K).

Many aspects of our wedge-based four-wave-mixing (FWM) apparatus, which is similar to a previous design, ${ }^{31}$ were described previously. ${ }^{10}$ Additional details specific to the measurements in this work are as follows. We placed an optically-flat, $3.2 \mathrm{~mm}$ thick, UV-fused-silica window in the white-light arm of the noncollinear optical parametric amplifier (NOPA), reducing the amplified bandwidth to about $70 \mathrm{~nm}$ as shown by the dashed line in Fig. 2(a). Then we adjusted prism and grating compressors to optimize compression of the amplified output pulse. ${ }^{56}$ The prism compressor used a Brewster angle UV-fused-silica prism (Newport 10SB10) in a single-prism configuration $^{57}$ with a distance of about $15 \mathrm{~cm}$ between the prism and the retroreflector (Edmund NT49-666), and the grating compressor used a ruled 600 lines $\mathrm{mm}^{-1}$ grating (Thorlabs GR25-0605) and a $10 \mathrm{~cm}$ focal-length cylindrical mirror (CVI PS-SCC-50.8-203.4-UV) oriented in a horizontally dispersed but vertically displaced 4-f configuration to minimize coupling between the spatial mode and the temporal profile. The pulse at the sample position was about $11 \mathrm{fs}$ in duration (intensity temporal FWHM), had a bandwidth of $61 \mathrm{THz}$ (intensity spectral FWHM), was characterized by a nearly featureless frequency-resolved optical gating (FROG) surface $^{58}$ (shown in Fig. 2(e)), and had no detectable angular dispersion.

The wedge calibration factors of approximately $26 \mathrm{fs} \mathrm{mm}^{-1}$ (known within $\pm 0.0001 \mathrm{fs}^{-1}$ ) were determined by a combination of two measurements. We first used a $594 \mathrm{~nm}$ $\mathrm{HeNe}$ source to determine initial calibration values (within

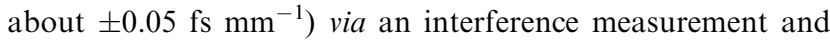
then found the more accurate values by removing any residual phase roll measured in a frequency-resolved heterodyne-detected transient-grating measurement of an appropriate laser dye. We checked the frequency dependence of the calibration factors by additional measurements using three other $\mathrm{CW}$ lasers at wavelengths of $532 \mathrm{~nm}, 632 \mathrm{~nm}$, and $730 \mathrm{~nm}$ to compile a table of frequency-dependent calibration values. For any given wedge, the value changes by about $0.2 \mathrm{fs} \mathrm{mm}^{-1}$ from 532 to $730 \mathrm{~nm}$. We illustrate the frequency-resolved heterodyne-detected transientgrating measurement in Fig. 2(f), where the phase roll has already been minimized. The visible oscillations are due to an intramolecular vibrational mode; the oscillations are not a result of imperfections in the translation stages. Since the stripes are essentially vertical across the spectrum, we conclude that the frequency-dependence of the wedge calibration factors is minimal for the bandwidth of the pulse. For the measurements in this work, we performed the frequency-resolved heterodyne-detected transient-grating measurement on the dye cresyl violet perchlorate solvated in methanol (optical density of about 0.1; both purchased and used as received from Sigma Aldrich) because 
it absorbs near $590 \mathrm{~nm} .{ }^{59}$ A neutral-density filter in the reference beam reduced its intensity by $10^{4}$ and was of appropriate thickness so that this pulse interacted with the sample about 250 fs before the final excitation field.

The 2D ES measurements involved a scanning time period $\tau_{1}$ from \pm 45 fs in 0.15 fs steps for each $\tau_{2}$ value, which was varied from 0 to $400 \mathrm{fs}$ in $5 \mathrm{fs}$ steps. Positive (negative) $\tau_{1}$ values indicate the rephasing (nonrephasing) pulse-timing sequence. These steps and bounds give numerical frequency resolutions of $1.7 \mathrm{THz}$ and $2.5 \mathrm{THz}$ in the absorption $\left(\nu_{1}\right)$ and $\nu_{2}$ dimensions, respectively. Zero-padding these two numerical dimensions decreases the frequency step size in the Fourier domain $(\Delta \nu)$, but does not improve the fundamental resolution. The spectral resolution of the emission dimension $\left(\nu_{3}\right)$ is limited by the grating/lens resolution of the spectrometer (about $1 \mathrm{~nm}$ ), which corresponds to a (nonlinear) frequency resolution below $1 \mathrm{THz}$ near $600 \mathrm{~nm}$.

Sample optical density at $585 \mathrm{~nm}$ was about 0.17 in a $1 \mathrm{~mm}$ pathlength flow cell (Starna Cells 48-Q-1). A peristaltic pump (Cole-Parmer Masterflex) flowed the sample at a rate of

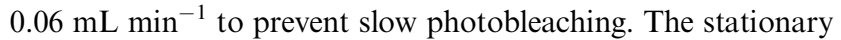
excitation beam (which contributes the final electric field) was chopped using a rotary beam chopper (Thorlabs MC200) to remove scatter contributions from the first two excitation beams. A charged-coupled-device (CCD) spectrometer (Andor SR-163 with a 600 lines $\mathrm{mm}^{-1}$ grating coupled to an Andor iDus DU401A-FI that has 1024 pixels in the spectrally dispersed dimension) set at $-60{ }^{\circ} \mathrm{C}$ was used to detect the emitted signal.

Before and after the 35 hours of time required to perform the ten 2D ES measurements of PC645, we measured reference 2D ES of the laser dye to detect any changes in alignment or power loss; the measurements were identical within the noise of the system. We performed four measurements of the $\mathrm{pH} 5.7$ sample and three each for the 6.5 and 7.4 samples, varying the measurement order randomly to reduce possible consequences of laser drift. In between each measurement we rinsed the sample cell and tubing using a large volume of distilled water. The extra scan of the $\mathrm{pH} 5.7$ sample was performed with triple the energy per beam (about $15 \mathrm{~nJ}$ pulse ${ }^{-1}$ ) compared to the other nine measurements (about $5 \mathrm{~nJ}$ pulse ${ }^{-1}$ ); it showed identical features and dynamics, albeit with larger amplitudes.

2D ES data analysis consisted of five steps. The first four steps executed the spectral interferometry algorithm, ${ }^{60}$ which involved mathematical manipulations of the emission dimension $\left(\nu_{3}\right)$ to generate the complex-valued data set, and the fifth step was Fourier transformation of the oscillations during the scanned dimension to generate the absorption dimension $\left(\nu_{1}\right)$. The analysis procedure did not involve spectral filters, apodization functions, or phase-flattening routines. We inspected the lineshapes of the separated rephasing and nonrephasing components before summing them to create the total 2D ES spectrum.

\section{Results}

In Fig. 3 we display representative 2D spectra from one measurement of the $\mathrm{pH} 6.5$ sample. Separated rephasing and nonrephasing components are presented in Appendix 1. The spectra in Fig. 3, which are the real part of the total complex-valued signal at the indicated $\tau_{2}$ values, contain several notable features.
There is a prominent cross peak located at an absorption frequency of about $520 \mathrm{THz}$ and an emission frequency of about $500 \mathrm{THz}$. There is a strong bleach component on the diagonal at about $520 \mathrm{THz}$ and additional smaller-amplitude bleach components throughout the surface. Throughout the scanned time window, the bleach surface is not symmetric about the diagonal. Ripples present in some spectra (the $\tau_{2}=75 \mathrm{fs}$ spectrum for example) are due to interference between the desired third-order nonlinear signal and a small amount of residual scatter. This could have been removed through filtering - an apodization function for example - but at the risk of losing the information content.

We first extracted the dynamics from the cross-peak location as indicated by the dashed lines in the $\tau_{2}=55 \mathrm{fs}$ spectrum in Fig. 3. We integrated the centre of the peak (519 THz, $499 \mathrm{THz})$ over a $\pm 1 \mathrm{THz}$ range in both dimensions. Other spectral features, the shoulder at $(470 \mathrm{THz}, 430 \mathrm{THz})$, for example, also contains dynamics, but in this work we focus mainly on the behaviour of the prominent cross peak.

In Fig. 4(a) we show the extractions from the real part of the total signal for the three different $\mathrm{pH}$ samples; the solid lines represent the mean and the error bars indicate one standard deviation $(\sigma)$ above and below the mean ( $2 \sigma$ total). The three traces are vertically offset for clarity. Given the similarity of the traces in Fig. 4(a), we averaged all of the data sets and plot the real and magnitude parts of the global mean in Fig. 4(b) and (c), respectively.

We fit the traces shown in Fig. 4(b) and (c) using a nonlinear least-squares method; the difference between the measured data (blue curves) and the fits (red curves) are the residuals (black curves). The residuals are plotted on the same scale as the traces but with a different zero offset for clarity. We excluded the values before $\tau_{2}=20 \mathrm{fs}$ because a non-negligible portion of the initial signal is due to nonresonant responses from the solvent and cell windows. The fit function was the sum of a constant (DC) background, a non-oscillatory exponential decay, and eight exponentially-decaying oscillatory terms (cosines). Tables 1 and 2 list the parameters used to generate the fits, where $95 \%$ confidence levels are noted and three dephasing times are excluded because the best fits contained (very low amplitude) non-decaying terms. Attempts to fit the data with fewer parameters resulted in residuals that were either outside the error bars of the measurement, contained obvious oscillations, or both. For example, removing the two lowest-amplitude oscillatory components (9.6 and $33 \mathrm{THz})$ resulted in a fit of the magnitude trace that showed disagreement in the two areas marked by the yellow boxes in Fig. 4(c) and whose residual showed clear low-frequency oscillations. The yellow boxes also highlight areas that, even with eight oscillatory components (35 total parameters), the fit to the mean is not perfect. We tested the eight-oscillatory-component fit function by seeding each data set with several arbitrary sets of initial parameters; in all cases the parameters converged to statistically similar values.

Total 2D ES signals are the sum of rephasing and nonrephasing components. In Fig. 5(a) we present the two components leading to the trace of the cross peak plotted in Fig. 4(b). Both contributions oscillate. The quality of the data and the strength of the oscillations indicate that Fourier analysis may be appropriate. 

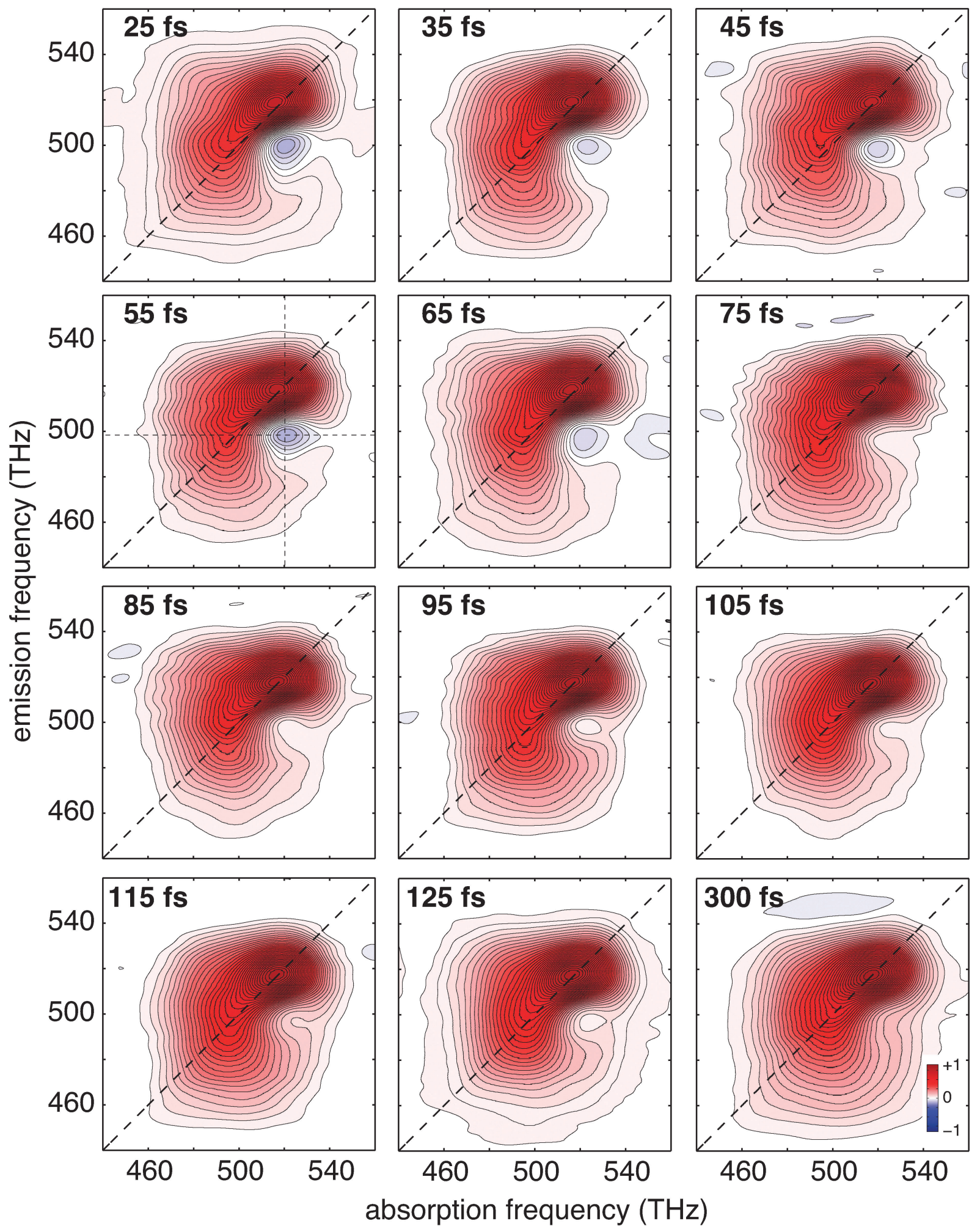

Fig. 3 Representative 2D spectra from one data set at $\mathrm{pH}$ 6.5. The spectra are the real part of the total complex-valued signal. Thin dashed vertical and horizontal lines in the $\tau_{2}=55 \mathrm{fs}$ spectrum indicate the cross-peak extraction coordinates. The spectra are individually normalized with 33 evenly spaced contours.

The resultant $\nu_{2}$ spectra for one measurement at $\mathrm{pH} 6.5$ are displayed in Fig. 5(b). The black (red) line is the spectral amplitude of the nonrephasing (rephasing) component of the Fourier transform of the real part of the time-domain trace. The blue arrows in
Fig. 5 highlight two important modes that we investigate further below. Although the $\tau_{2}$ time step sets the Nyquist limit at $100 \mathrm{THz}$, the optical bandwidth restricts the high-frequency limit to about $60 \mathrm{THz}$. We discuss this further in the Discussion section. 
(a)

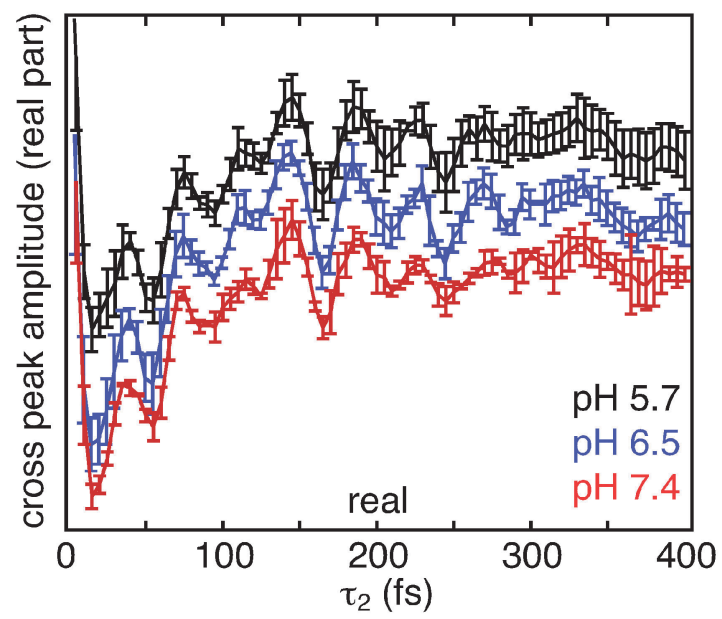

(b)

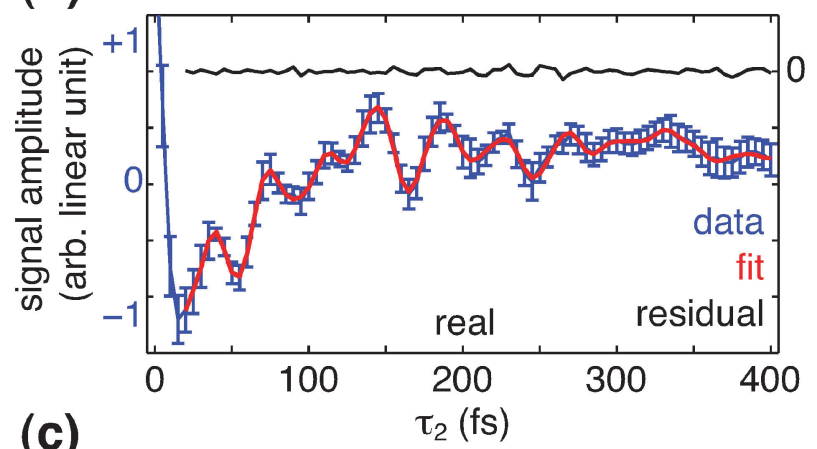

(c)

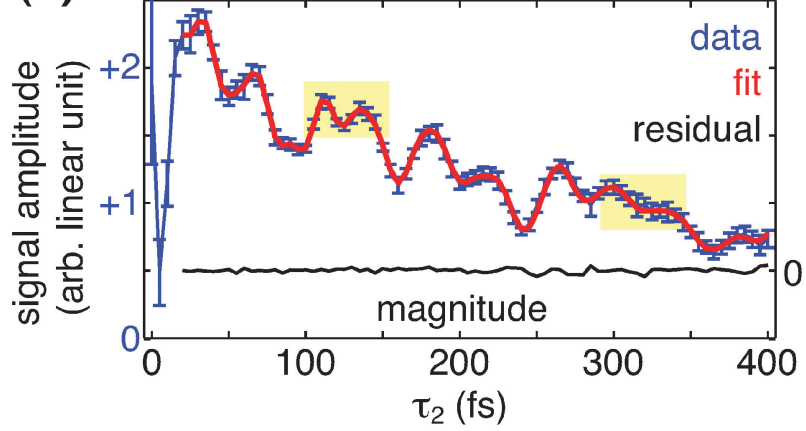

Fig. 4 Coherent dynamics in PC645. (a) Cross-peak extractions from the real part of the total signal show coherent oscillations during $\tau_{2}$ for all $\mathrm{pH}$ values. Traces are offset vertically for clarity. (b) Real part of the average of all ten 2D ES measurements. (c) Magnitude of the average of all ten 2D ES measurements. The difference between the fits (red curves) and the data (blue curves) are the residuals (black curves). As described in the text, the yellow boxes highlight areas that were difficult to fit using fewer than eight oscillatory components. In all panels, error bars indicate one standard deviation.

To investigate the two highlighted modes quantitatively, we generated plots similar to Fig. 5(b) for the other nine 2D ES data sets. In each $\nu_{2}$ spectrum, we estimated the signal and noise levels for the rephasing and nonrephasing components of the 21 and $26 \mathrm{THz}$ modes. Statistical analysis of the results is presented in Table 3, where the error values are one standard deviation of the ten measurements. We did not fit or estimate the peak frequencies due to the aforementioned fundamental $\nu_{2}$ resolution.

A set of $2 \mathrm{D}$ spectra can be stitched together to construct a complex-valued $3 \mathrm{D}$ data set in the $\left(\nu_{1}, \tau_{2}, \nu_{3}\right)$ domain and then
Table 1 Parameters (with 95\% confidence intervals) used in the fit presented in Fig. 4(b)

\begin{tabular}{llll}
\hline Frequency/THz & Dephasing/fs & Amplitude/arb. & Phase/rad \\
\hline- & $\overline{-}$ & $0.33 \pm 0.01$ & - \\
$\overline{6}$ & $40 \pm 10$ & $-2.1 \pm 0.83$ & - \\
$8.5 \pm 0.8$ & $110 \pm 40$ & $0.57 \pm 0.59$ & $0.3 \pm 0.5$ \\
$14.9 \pm 0.3$ & - & $0.08 \pm 0.04$ & $1.7 \pm 2.2$ \\
$21.4 \pm 0.4$ & $180 \pm 110$ & $0.30 \pm 0.11$ & $-0.1 \pm 0.3$ \\
$25.9 \pm 0.2$ & $140 \pm 30$ & $0.44 \pm 0.16$ & $0.5 \pm 0.4$ \\
$33.4 \pm 0.9$ & - & $0.60 \pm 0.13$ & $0.9 \pm 0.2$ \\
$40.5 \pm 5.3$ & $20 \pm 10$ & $0.02 \pm 0.01$ & $0.7 \pm 1.4$ \\
$50.1 \pm 1.2$ & $370 \pm 20$ & $1.2 \pm 2.3$ & $1.1 \pm 1.1$ \\
\hline
\end{tabular}

Table 2 Parameters (with 95\% confidence intervals) used in the fit presented in Fig. 4(c)

\begin{tabular}{llll}
\hline Frequency/THz & Dephasing/fs & Amplitude/arb. & Phase/rad \\
\hline- & - & $0.38 \pm 0.19$ & - \\
$\overline{7}$ & $260 \pm 50$ & $1.84 \pm 0.11$ & - \\
$9.4 \pm 1.5$ & - & $0.11 \pm 0.14$ & $-1.0 \pm 2.3$ \\
$13.9 \pm 0.3$ & $480 \pm 450$ & $0.06 \pm 0.46$ & $0.0 \pm 2.1$ \\
$21.1 \pm 0.3$ & $230 \pm 90$ & $0.24 \pm 0.12$ & $1.7 \pm 0.5$ \\
$25.6 \pm 0.1$ & $300 \pm 60$ & $0.23 \pm 0.08$ & $2.0 \pm 0.2$ \\
$33.3 \pm 0.5$ & $320 \pm 160$ & $0.25 \pm 0.05$ & $2.0 \pm 0.2$ \\
$41.7 \pm 1.6$ & $50 \pm 16$ & $0.06 \pm 0.04$ & $1.4 \pm 0.6$ \\
$51.1 \pm 0.9$ & $90 \pm 30$ & $0.14 \pm 0.19$ & $1.8 \pm 0.7$ \\
\hline
\end{tabular}
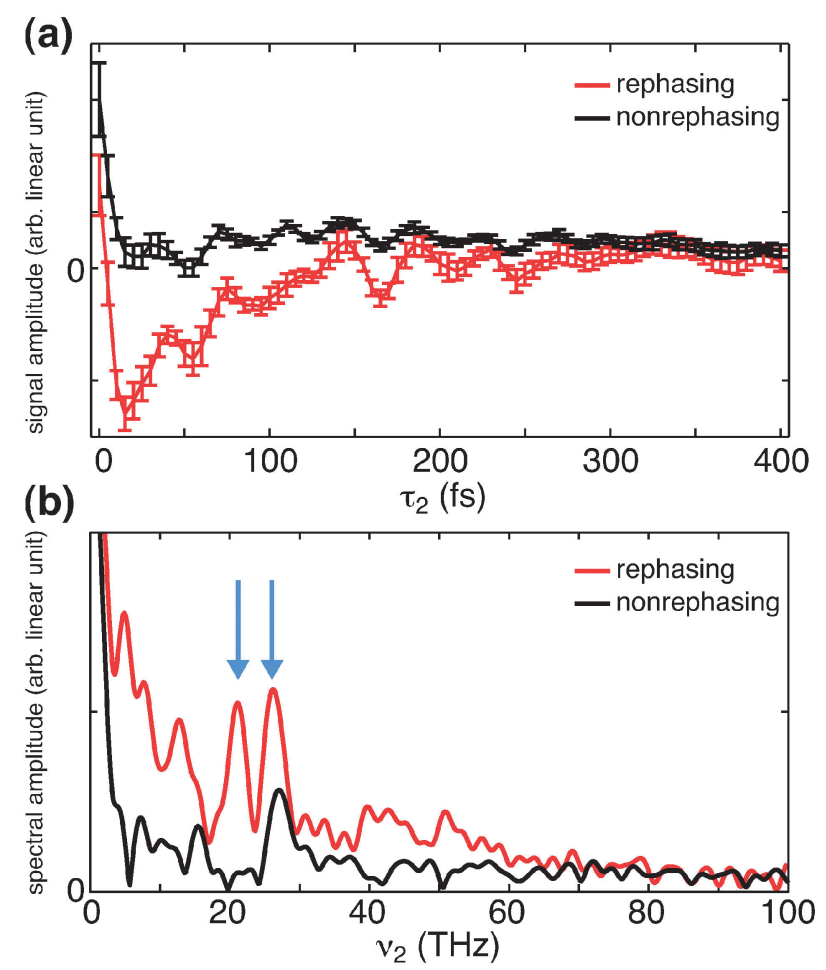

Fig. 5 Comparison of rephasing (red) and nonrephasing (black) contributions. (a) Mean and one standard deviation of the two components of the global average trace presented in Fig. 4(b). (b) Fourier transformation of the coherent oscillations during time period $\tau_{2}$ leads to spectra from one representative measurement. Blue arrows highlight two large-amplitude peaks at about $21 \mathrm{THz}$ and $26 \mathrm{THz}$.

Fourier transformed across time period $\tau_{2}$ to create a $3 \mathrm{D}$ spectral solid ${ }^{61}$ after modest zero padding in the $\tau_{2}$ dimension. 
Table 3 Signal-to-noise of the two peaks highlighted in Fig. 5. A S/N value of 1 indicates that the peak is indistinguishable from noise

\begin{tabular}{lll}
\hline Frequency/THz & Rephasing S/N & Nonrephasing S/N \\
\hline 21 & $2.4 \pm 0.4$ & $1.2 \pm 0.3$ \\
26 & $2.6 \pm 0.5$ & $2.7 \pm 0.7$ \\
\hline
\end{tabular}

Here we do not display the spectral solid itself because it is difficult to view and the features at $\nu_{2}=0$ dominate the spectrum. (The seemingly bright feature at $\nu_{2}=26 \mathrm{THz}$ has an amplitude about 20 times lower than the $0 \mathrm{THz}$ feature.) In Fig. 6 we present several selectively integrated sections (projections) of the spectral solid. Another consequence of the large amplitude of the $0 \mathrm{THz}$ mode is that we individually normalized the presented spectra. Normalization values, relative to the $0 \mathrm{THz}$ projection, are indicated in each spectrum. The yellow arrows in Fig. 6 highlight three important locations where certain modes are maximized. In other words, certain $\nu_{2}$ frequencies are more prominent in some locations of the 2D spectrum than other $\nu_{2}$ frequencies. The negative amplitudes of some modes indicate that they are due to excited-state absorption (ESA) pathways. Since the main cross peak is below the diagonal, where absorption frequencies are greater than emission frequencies, the $\nu_{2}$ frequencies often have negative values. This is merely a result of the frequency-accounting scheme used in the nonlinear optical spectroscopy formalism and has been discussed previously in the context of $\nu_{2}$ frequencies in $2 \mathrm{D} \mathrm{ES}{ }^{61,62}$

Certain projections of the spectral solid are noteworthy. The two projections at positive $\nu_{2}$ frequencies are roughly the noise spectrum of the system and therefore essentially reproduce the pulse spectrum symmetrically about the diagonal. Similarly, the projection at $-90 \mathrm{THz}$ is also essentially noise. The $-50 \mathrm{THz}$ projection contains several distinct peaks along the diagonal and a significant positive-amplitude cross peak centred at $(525 \mathrm{THz}, 485 \mathrm{THz})$. The -40 and $-30 \mathrm{THz}$ projections are similar to the $-50 \mathrm{THz}$ projection although the location of the cross peak shifts slightly and there is an increase in the amplitude of the low emission-frequency ESA peak. On the other hand, the $-26 \mathrm{THz}$ projection contains a positive-amplitude cross peak located at $(520 \mathrm{THz}, 500 \mathrm{THz})$, a significantly different location than the cross peak in the $-50 \mathrm{THz}$ projection. The $-21 \mathrm{THz}$ projection contains this new cross peak - at slightly shifted coordinates of $(524 \mathrm{THz}$, $499 \mathrm{THz}$ - and also contains a negative-amplitude cross peak very near the diagonal. These two peaks trade relative amplitudes by the $-7 \mathrm{THz}$ projection, where the negative-amplitude peak is centred at $(519 \mathrm{THz}, 508 \mathrm{THz})$. In all of the projections from -90 to $-7 \mathrm{THz}$, there is an ESA feature located at $(510 \mathrm{THz}$, $473 \mathrm{THz}$ ), as indicated in the $-13 \mathrm{THz}$ projection.

The $0 \mathrm{THz}$ projection contains mostly positive-amplitude features, although one ESA peak is present in the cross peak location of $(520 \mathrm{THz}, 501 \mathrm{THz})$. The positive-amplitude bleach surface is very asymmetric.

Given the highlighted peaks in Fig. 5(b), we focus on the $-21 \mathrm{THz}$ and $-26 \mathrm{THz}$ projections. Two cross peaks appear in each projection. In the $-26 \mathrm{THz}$ projection, the major cross peak is located at $(521 \mathrm{THz}, 499 \mathrm{THz})$, while in the $-21 \mathrm{THz}$ projection, it is located at $(525 \mathrm{THz}, 498 \mathrm{THz})$. The second, smaller-amplitude cross peak is located at $(517 \mathrm{THz}, 510 \mathrm{THz})$ in both projections, but has a positive amplitude in the $-26 \mathrm{THz}$ projection and a negative amplitude in the $-21 \mathrm{THz}$ projection. Both projections contain the strong low emission-frequency ESA signal, and have bleach surfaces similar to the $0 \mathrm{THz}$ projection.

Finally, we display line-outs from the 3D spectral solid in Fig. 7. A line-out is the $\nu_{2}$ spectrum at certain absorption and emission coordinates, the spectral version of the time-domain traces shown in Fig. 4. The five panels are $\pm 1 \mathrm{THz}$ integrations about the indicated coordinates. There are several noteworthy features in these line-out spectra. First of all, in the $(526 \mathrm{THz}$, $494 \mathrm{THz}$ ) line-out (violet), peaks appear at all of the highfrequency modes found in the fit presented in Tables 1 and 2. Specifically, distinct peaks appear at $-21 \mathrm{THz},-26 \mathrm{THz}$, $-40 \mathrm{THz}$, and $-50 \mathrm{THz}$. There may also be low-frequency modes present closer to $0 \mathrm{THz}$, but they are difficult to discern from the tail of the main peak.

At a slightly different location in the cross peak $(519 \mathrm{THz}$, $501 \mathrm{THz}$ (green line-out), the high-frequency modes greater than $-30 \mathrm{THz}$ have diminished somewhat relative to the still prominent peaks at -21 and $-26 \mathrm{THz}$. This is in accord with the selective integrations presented in Fig. 6. Although the $-15 \mathrm{THz}$ mode seems very prominent in this line-out, this may be serendipitous. As discussed below, it is significant that both the violet and green line-outs have peaks at both positive and negative $26 \mathrm{THz}$.

The $(516 \mathrm{THz}, 511 \mathrm{THz})$ line-out (orange) is very near the diagonal and contains little notable signal other than the $0 \mathrm{THz}$ component. This serves as a reference so that identification of peaks in the other line-outs is understandable.

The blue line-out is taken from where the prominent ESA signal appeared in the $-7 \mathrm{THz}$ projection of the spectral solid in Fig. 6, at $(510 \mathrm{THz}, 473 \mathrm{THz})$. The $0 \mathrm{THz}$ component has lost significant amplitude on the negative-frequency side. The peak at $-21 \mathrm{THz}$ seems to have disappeared; the peaks at -26 and $-40 \mathrm{THz}$ are still prominent and have positive amplitude; and the peak near $-50 \mathrm{THz}$ has a large amplitude.

Finally, the black line-out shows the frequencies present at (475 THz, $525 \mathrm{THz}$ ), where we noted above when describing the data presented in Fig. 3 that there seems to be an oscillatory component of the shoulder. Since this line-out is for a peak located on the opposite side of the diagonal, $\nu_{2}$ frequency values are positive. We observe a sharp feature at about $50 \mathrm{THz}$, which corresponds to the difference between the absorption and emission frequencies, and also a rise toward the end of the spectrum at $\nu_{2}$ frequencies of about 80-100 THz, which would correspond to the periodicity of the oscillatory shoulder in Fig. 3.

\section{Discussion}

\section{1 pH dependence}

The three $\mathrm{pH}$ levels were chosen to span the range of physiological $\mathrm{pH}$ changes within the algal lumen. ${ }^{45}$ Linear spectra at lower $\mathrm{pH}$ levels have also been measured. ${ }^{55,63}$ Cryptophyte algae are the only known photosynthetic organisms in which the light-harvesting antennae proteins are bathed in the $\mathrm{pH}$-changing environment of the lumen. ${ }^{64}$ Thus we investigated whether the $\mathrm{pH}$ drop regulates the initial dynamical response, in addition to triggering the fluorescence-quenching mechanism. Previous $\mathrm{pH}$-dependent 

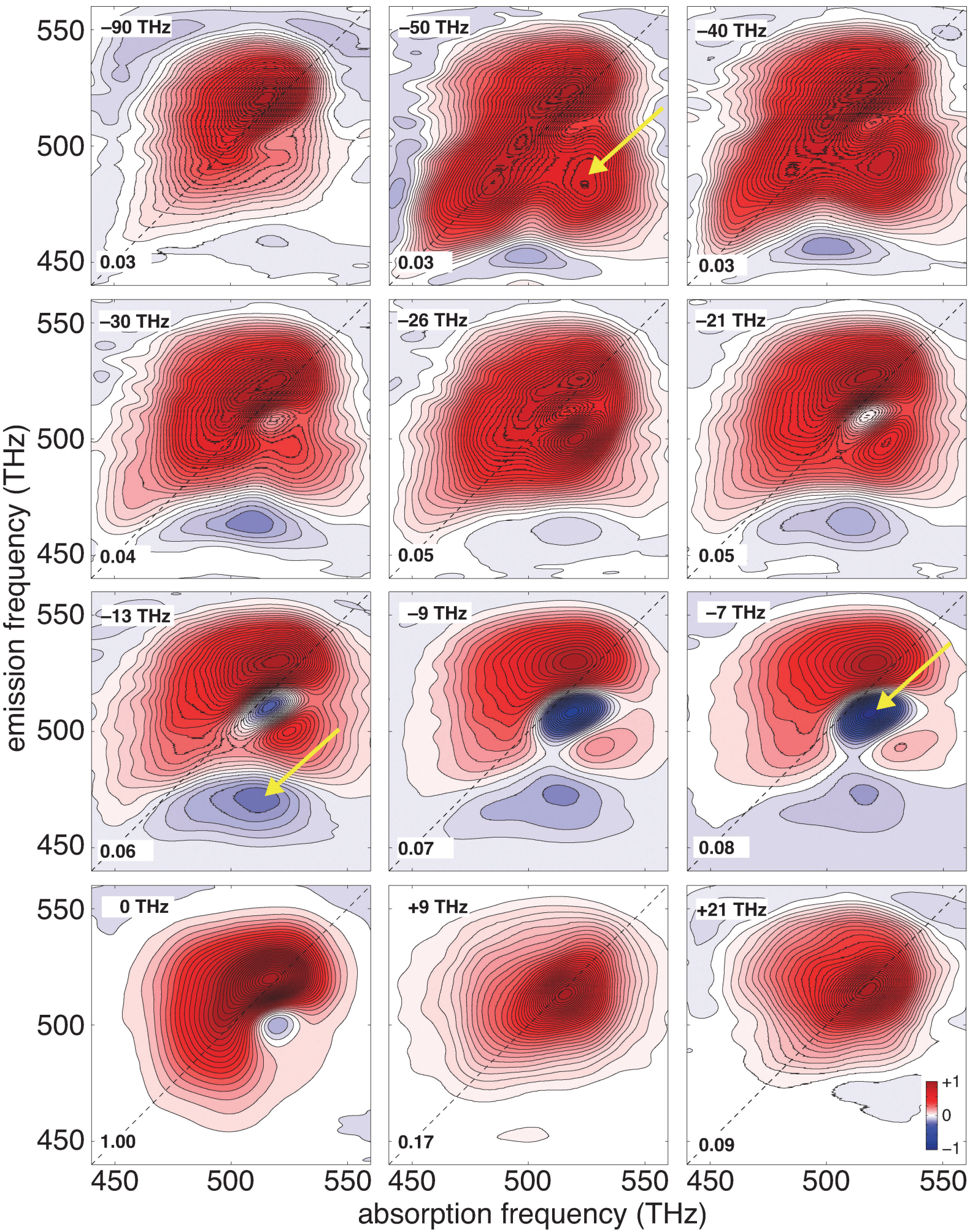

Fig. 6 Selectively integrated planes of the 3D spectral solid from one measurement at $\mathrm{pH}$ 6.5. Integrations were performed about the indicated $\nu_{2}$ frequencies within a $\pm 1 \mathrm{THz}$ range. Yellow arrows indicate prominent cross peaks. Values in the bottom left corner of each spectrum indicate amplitudes relative to the $0 \mathrm{THz}$ projection. One way to interpret these spectra is as follows, using the $-40 \mathrm{THz}$ projection as an example. There are three significant portions of the $2 \mathrm{D}$ spectrum that oscillate with a frequency of $-40 \mathrm{THz}$, the diagonal peaks at $525 \mathrm{THz}$ and $490 \mathrm{THz}$ and the cross peak at $(525 \mathrm{THz}, 490 \mathrm{THz})$.

Raman studies of the vibrational modes of similar proteins and chromophores have shown small frequency shifts (about $15 \mathrm{~cm}^{-1}$ ) as the $\mathrm{pH}$ is lowered. ${ }^{65-67}$ However, these shifts are smaller than the $\nu_{2}$ frequency resolution in the 2D ES measurements presented here. Electronic dynamics hinge on the molecular electronic states, their couplings, and their relative orientations. ${ }^{52,68}$ 

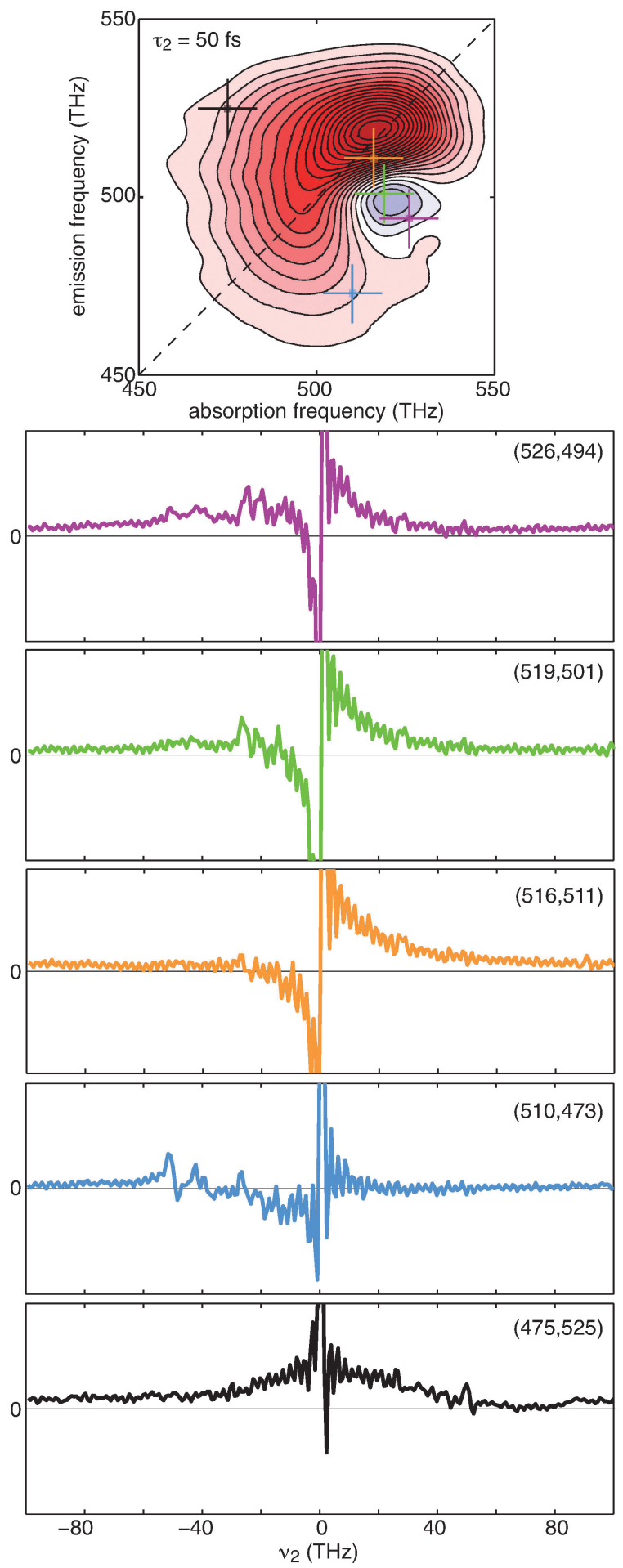

Fig. 7 Line-outs of the 3D spectral solid at the indicated (absorption, emission) coordinates, given in $\mathrm{THz}$ units. Integration was performed in a $\pm 1 \mathrm{THz}$ region.

As shown in Fig. 2(a)-(d), the linear absorption, fluorescence, and CD spectra do not appreciably change as a function of $\mathrm{pH}$. The lack of variation in the spectra indicates that the underlying chromophore electronic states and couplings do not change with the $\mathrm{pH}$ drop. Similarly, the lack of change in the CD spectra indicates that the secondary structure of the protein has also not been altered significantly by the $\mathrm{pH}$ variation. Although the fluorescence spectra did show reproducible $\mathrm{pH}$ dependence on the intensity of the signal, the variation in the $\mathrm{pH}$ dependence prevented even a qualitative analysis.

Thus it is perhaps not surprising that the traces presented in Fig. 4(a) show that the $\mathrm{pH}$ drop does not alter the initial dynamics. The protein environment appears to protect the electronic states and their couplings, and it therefore protects the coherence. We conclude that the phycobiliprotein PC645 is rather robust and unaffected by this dramatic change in the $\mathrm{pH}$ level. This conclusion should stimulate further interest into the question of why the light-harvesting proteins migrated into the lumen. ${ }^{64}$ It may be the case that the proteins exist in the lumen simply because they do not have an attachment apparatus such as the one used by phycobilisomes.

\subsection{Signal oscillations}

Using time-domain fit functions, we found that no fewer than eight oscillatory components were required to fit the dynamics to within the error of the measurement. Often a point of confusion is how there can be multiple frequencies underlying the dynamics at a particular location in the 2D spectrum. A cursory inspection of the relevant double-sided Feynman diagrams (see Appendix 2) suggests that for most locations in the $2 \mathrm{D}$ spectrum, there should be only one frequency during time period $\tau_{2}$, and this frequency should be given by the difference between the absorption and emission frequencies. However, an alternative interpretation is that the absorption and emission frequencies set which specific element of the density matrix is probed, but the dynamics during $\tau_{2}$ are given by the time dependence of the full density matrix, which is governed by a set of coupled differential equations.

We must also consider the situations when multiple absorption features overlap or the additional vibronic cross peaks described in Appendix 2. Oscillations at one specific location in the $2 \mathrm{D}$ spectrum can thus appear at frequencies other than (but certainly including) the difference between the absorption and emission frequencies. As described below, this becomes useful when inspecting slices of the spectral solid.

The time-domain oscillations, which were extracted from coordinates whose difference frequency was $20 \mathrm{THz}$, had particularly strong contributions from the 21 and $26 \mathrm{THz}$ modes. These two modes had well-defined frequencies, dephasing times, amplitudes, and phases in the fits as evidenced by agreement between the values in Tables 1 and 2 and their small confidence intervals. Unsurprisingly, the phase values are different between the real and magnitude data sets. Although the dephasing value of the $26 \mathrm{THz}$ mode differs by a factor of almost two (well beyond the confidence interval) the amplitudes and frequencies for both modes - and the dephasing value for the $21 \mathrm{THz}$ mode - fall within statistically similar confidence intervals. In contrast, the parameters of the three lowest-amplitude modes $(9,33$, and $50 \mathrm{THz})$ had larger confidence intervals. The frequencies were statistically similar between the two data sets, but the dephasing, amplitude, and phase values all differed significantly. The remaining three modes $(7,14$, and $40 \mathrm{THz})$ fall somewhere between these two bounds in terms of both the agreement between the real and magnitude fits and the size of the confidence intervals. 
Again the frequencies were similar and the other values were less consistent.

As expected, there is a strong correspondence between the frequencies found by the time-domain fit functions and the location of peaks in the Fourier transforms presented in Fig. 5(b), 6, and 7. For example, the -50 and $-40 \mathrm{THz}$ projections of the $3 \mathrm{D}$ solid contain very clear cross peaks whose shoulders overlap with the location of the time-domain extraction position. We attempt to assign several of the eight modes below using information from both the time and frequency domains.

\subsection{Assignment of oscillations}

Recent interest in quantum-coherent dynamics has been based on the expectation that the cross-peak oscillations were signatures of electronic coherences and not signatures of vibrational coherences (intramolecular nuclear wavepackets oscillating on either the excited or ground electronic potentials). Numerous recent studies ${ }^{35-42,69}$ have pointed out that using 2D ES to distinguish between vibrational and electronic coherences can be challenging because their spectral signatures are very similar.

In previous work, ${ }^{10}$ we demonstrated that separation of the rephasing and nonrephasing components is useful for distinguishing between vibrational and electronic coherences in 2D ES because the complete lack of oscillations in the nonrephasing component indicates that the coherence is due to electronic coupling. We tested the differentiation protocol-and found that it works as anticipated - on systems which are known to have only one kind of physical process which could lead to cross-peak oscillations: GaAs quantum wells, ${ }^{70} \mathrm{CdSe}$ nanocrystals, ${ }^{71}$ and the laser dyes Rhodamine $6 \mathrm{G}$, Rhodamine 101 , and cresyl violet perchlorate. ${ }^{10}$ In previous work ${ }^{10}$ we analyzed the dynamics of the prominent cross peak in the 2D ES of the PC645 light-harvesting complex from the cryptophyte Chroomonas sp. CCMP270 for the first $100 \mathrm{fs}$ of time period $\tau_{2}$ at a $\mathrm{pH}$ of 6.8 , and we noted that the test for a complete lack of oscillations can often be inconclusive when examining only time-domain traces.

Here, using a detailed study of the nonlinear response functions along with quantitative analysis in both the time and frequency domains of the signal measured to $400 \mathrm{fs}$, we resolve much of the ambiguity we previously encountered. By inspecting the complete set of double-sided Feynman diagrams depicted in Appendix 2 for the anticipated model system having four energy levels, we also identify two additional ways to differentiate between vibrational and electronic coherences.

As we pointed out previously, the key to differentiating electronic from vibrational coherences is the energy-level structure, see Fig. 1 in ref. 10. Compared to the three-level, 'V-shaped' system often used to describe the minimal model for electronic coherence, the fourth state in the vibrational model not only leads to oscillations in the nonrephasing component of the cross peak, but it also leads to two extra cross peaks below the diagonal (at emission energy of $e-\nu$ ) and a phase shift in the 'normal' below-diagonal cross peak (oscillations at both $+\nu$ and $-\nu$ instead of only $-\nu$ ). The two extra cross peaks are a useful diagnostic if the pulse bandwidth has sufficient spectral brightness on the low-energy side of the main absorption feature. The phase shift is a useful diagnostic if the phase of the oscillations is well defined or if Fourier-transform techniques have sufficient signal-to-noise. Below we apply two of the differentiation protocols (the nonrephasing oscillations and phase shift) to the two modes - the 21 and $26 \mathrm{THz}$ modes - that were of both sufficient amplitude and relatively low background.

For the purpose of discussion we separate the eight modes into two groups - those frequencies higher or lower than $20 \mathrm{THz}$ - and then compare the 2D ES results above to previous Raman results ${ }^{65-67,72-77}$ and to $1 \mathrm{D}$ nonlinear spectroscopy results ${ }^{78-80}$ to assign some of the eight modes. The Raman studies - which report on intramolecular vibrational modes - of other protein-bound open-chain tetrapyrrole chromophores revealed a manifold of vibrational modes between 600 and $1800 \mathrm{~cm}^{-1}$. Peaks occur at about $670 \mathrm{~cm}^{-1}, 820 \mathrm{~cm}^{-1}$, $1100 \mathrm{~cm}^{-1}, 1310 \mathrm{~cm}^{-1}$, and $1650 \mathrm{~cm}^{-1}$ but the frequencies and amplitudes can vary depending on which chromophore or protein is measured. The best comparison would be to a resonant-Raman measurement on the exact protein used here, but such a measurement has not yet been performed.

We begin with the high-frequency modes of $33 \mathrm{THz}$ $\left(1089 \mathrm{~cm}^{-1}\right), 40 \mathrm{THz}\left(1320 \mathrm{~cm}^{-1}\right)$, and $50 \mathrm{THz}\left(1650 \mathrm{~cm}^{-1}\right)$. These three modes were found in the time-domain fit and can be observed in the $\nu_{2}$ spectra taken from the cross-peak coordinates shown in violet and blue in Fig. 7. Their signatures can also be observed in the projections of the spectral solid in Fig. 6. Because the amplitudes of these three modes were small, it was not possible to perform quantitative analysis of the separated rephasing and nonrephasing components. Nevertheless, since these three modes closely match the $1100 \mathrm{~cm}^{-1}, 1310 \mathrm{~cm}^{-1}$, and $1650 \mathrm{~cm}^{-1}$ peaks present in the Raman spectra, it is likely that they are signatures of vibrational modes of one or more of the eight underlying chromophores.

It is apparent from the data shown in Fig. 5(a) that both the nonrephasing and rephasing contributions to the total cross-peak signal oscillate. Therefore at least a portion of the oscillating signal is due to vibrational coherences. The Fourier analysis of the separated components presented in Fig. 5(b) and Table 3 leads to a better understanding of the oscillations. The $26 \mathrm{THz}\left(845 \mathrm{~cm}^{-1}\right)$ mode is present in both rephasing and nonrephasing components, indicating that it is unambiguously a signature of vibrational coherence; the mode corresponds to the $820 \mathrm{~cm}^{-1}$ vibrational mode observed in the Raman studies.

On the other hand, only the rephasing component of the $21 \mathrm{THz}\left(696 \mathrm{~cm}^{-1}\right)$ mode is statistically distinguishable from noise. The differentiation protocol indicates that this mode is due to electronic coupling because there was no contribution from the nonrephasing component of the signal. Moreover, inspecting the violet, green, and orange line-outs in Fig. 7, it is clear that the $26 \mathrm{THz}$ mode is present at both positive and negative $\nu_{2}$ values but the $21 \mathrm{THz}$ mode is only present at negative $\nu_{2}$ values. Following Appendix 2, this is additional evidence that the $26 \mathrm{THz}$ mode is due to vibrational coherence but the $21 \mathrm{THz}$ mode is due to electronic coherence.

It is difficult to determine which exciton states are in superposition to create the electronic coherence because such an assignment requires a firm understanding of the exciton energies. In PC645 there are eight chromophore molecules 
with independent energies (often called site energies), some of which are thought to be very similar. Although a Hamiltonian has been proposed for PC $645,{ }^{16}$ it was based only on simulations of steady-state spectra ${ }^{68}$ (linear absorption, fluorescence, $\mathrm{CD}$, etc.). The procedure used to find this Hamiltonian did not incorporate simulations of time-domain nonlinear measurements. One study on a related phycobiliprotein ${ }^{81}$ found that multiple Hamiltonians can lead to sufficient fits to steady-state spectra. That study then used simulations of time-domain nonlinear measurements such as transient-absorption to refine the Hamiltonian. A study following the work in ref. 81 is currently underway to refine the PC645 Hamiltonian.

Nevertheless, here we use the proposed Hamiltonian ${ }^{16,68}$ — which has four excitons with frequencies above $480 \mathrm{THz}$ - as a starting point for assignment. The excitons at $529 \mathrm{THz}$ and $510 \mathrm{THz}$ originate mostly from a pair of dihydrobiliverdin (DBV) chromophores; the higher-energy (lower-energy) exciton is often noted as $\mathrm{DBV}_{+}\left(\mathrm{DBV}_{-}\right)$. The excitons at $498 \mathrm{THz}$ and $496 \mathrm{THz}$ originate mostly from a pair of mesobiliverdin (MBV) chromophores; the higher-energy (lower-energy) exciton is often noted as $\mathrm{MBV}_{B}\left(\mathrm{MBV}_{A}\right)$. The location of the cross peak in the time-domain data-absorption at $519 \mathrm{THz}$ and emission at $499 \mathrm{THz}$ - and in the frequency-domain data - absorption at $524 \mathrm{THz}$ and emission at $499 \mathrm{THz}$ - suggests a coherence between the $\mathrm{DBV}_{+}$and $\mathrm{MBV}_{B}$ excitons, which we suggested in previous work. ${ }^{10}$ However, a superposition between those two excitons would oscillate with a frequency of $31 \mathrm{THz}$, but the oscillation frequency found in both the time-domain fit and the frequency-domain spectrum in Fig. 5 is $21 \mathrm{THz}$. The two excitons whose difference frequency most closely corresponds to $21 \mathrm{THz}$ are the $\mathrm{DBV}_{+}$and $\mathrm{DBV}_{-}$excitons. Thus, we have encountered two problems in our attempt to determine which excitons are in superposition. The first is that the exciton energies are not known with sufficient accuracy; this can be ameliorated by finding a Hamiltonian that can lead to satisfactory fits of both the steady-state and time-domain nonlinear spectra. The second is that while the coordinates of the cross peak suggest a coherent superposition between the $\mathrm{DBV}_{+}$and $\mathrm{MBV}_{B}$ excitons, the oscillation frequency suggests a coherent superposition between the $\mathrm{DBV}_{+}$and $\mathrm{DBV}_{-}$excitons.

The dephasing time of the electronic coherence has also been of interest. ${ }^{5,9,82}$ Here we found the dephasing time of the electronic coherence to be $150 \pm 50 \mathrm{fs}$ at $298 \mathrm{~K}$, by taking the average of the dephasing values for this coherence from Tables 1 and 2. It would be fruitful to compare the dephasing time of the exciton-exciton coherence (meaning the electronic coherence) to the dephasing time of a ground-state-exciton coherence experimentally, since these two values have been compared theoretically. ${ }^{82}$ Here we measured the exciton-exciton coherence dephasing time to be $150 \pm 50 \mathrm{fs}$, but it is challenging to measure the ground-state-exciton coherence dephasing time. In principle it should be possible to extract the ground-state-exciton coherence dephasing time from the linewidth of the features in a linear absorption spectrum. However, the spectrum contains eight overlapping features, and, more importantly, it is known from low-temperature steady-state spectral studies of PC645 that the linewidth is dominated by inhomogeneous broadening. . $^{1,68}$ Therefore, it is currently difficult to extract the groundstate-exciton coherence dephasing time from experiments.
Nevertheless, it seems likely that this coherence has a dephasing time faster than $150 \mathrm{fs}$. If that is the case, more work is required to understand why the exciton-exciton coherence dephasing time is longer than the ground-state-exciton dephasing time. ${ }^{82}$ Clearly, much remains to be understood about the electronic coherence.

Returning to the components of the time-domain fit, the $13 \mathrm{THz}\left(430 \mathrm{~cm}^{-1}\right), 9 \mathrm{THz}\left(297 \mathrm{~cm}^{-1}\right)$, and $7 \mathrm{THz}\left(230 \mathrm{~cm}^{-1}\right)$ modes were outside the detection window of previous Raman studies and thus we are unable to compare our results to that technique. Moreover, the low-frequency modes were too low in amplitude in the 2D ES analysis (relative to the signal from the non-oscillatory background) to attribute to either vibrational or electronic coupling. Projections of the 3D spectral solid indicate that the signals originate from ESA pathways and thus knowledge of the higher-lying electronic states is needed for a complete analysis.

Our protocols assign the $21 \mathrm{THz}$ oscillations to electronic coherence. However, a peak near this frequency has been noted in the (nonresonant) Raman measurements. Quantumchemical computations of the Raman spectra are less successful at computing the presence of this mode ${ }^{77}$ versus the higherfrequency modes. It is possible that the $670 \mathrm{~cm}^{-1}$ peak in the Raman spectrum was not due to a chromophore vibrational mode but was instead a response of the amino acids since many amino acids contain strong Raman responses very near this frequency. Alanine, for example, which is by far the most abundant amino acid in PC645, has a strong feature at about $660 \mathrm{~cm}^{-1}$. 2D ES measurements performed with femtosecond pulses in the visible region of the spectrum only probe vibrations that are coupled strongly to the electronic excited states of the bilin chromophore.

Finally, we consider the possibility that both the electronic and vibrational modes need to be considered when discussing electronic coupling of organic molecules. Indeed, the models upon which we have based the differentiation protocols do not explicitly address this case, although it seems sensible that any vibronic modulation will lead to excited vibrational levels in a ground state (which would then lead to oscillations in the nonrephasing signal). On the other hand, studies on J-aggregate ${ }^{83-85}$ show that the electronic peaks do not contain vibronic modulations that are present in the individual chromophore (monomer). Future studies will investigate this question in more detail.

\subsection{Spectral solid extractions}

The extractions from the 3D spectral solid ${ }^{61,86}$ provide additional insights into the dynamics. To understand the extractions and line-outs of the 3D spectral solid as presented in Fig. 6 and 7, one must keep six points in mind. First, the fundamental $\nu_{2}$ resolution $\left(\Delta \nu_{2}\right)$ is given by the $\tau_{2}$ time step $\left(\Delta \tau_{2}\right)$ and the number of points $(N)$, such that $\Delta \nu_{2}=1 /\left(N \times \Delta \tau_{2}\right)$. Second, the bandwidth restricts the size of the window available to the absorption and emission dimensions, which will often truncate features. Third, negative-amplitude features in the real part of the spectral solid are due to ESA pathways. Fourth, the nonresonant response at $\tau_{2}=0 \mathrm{fs}$ in the time domain leads to a strong feature centred at $\nu_{2}=0$ in the frequency domain that has a nonzero tail out to about $20 \mathrm{THz}$ as evidenced in 

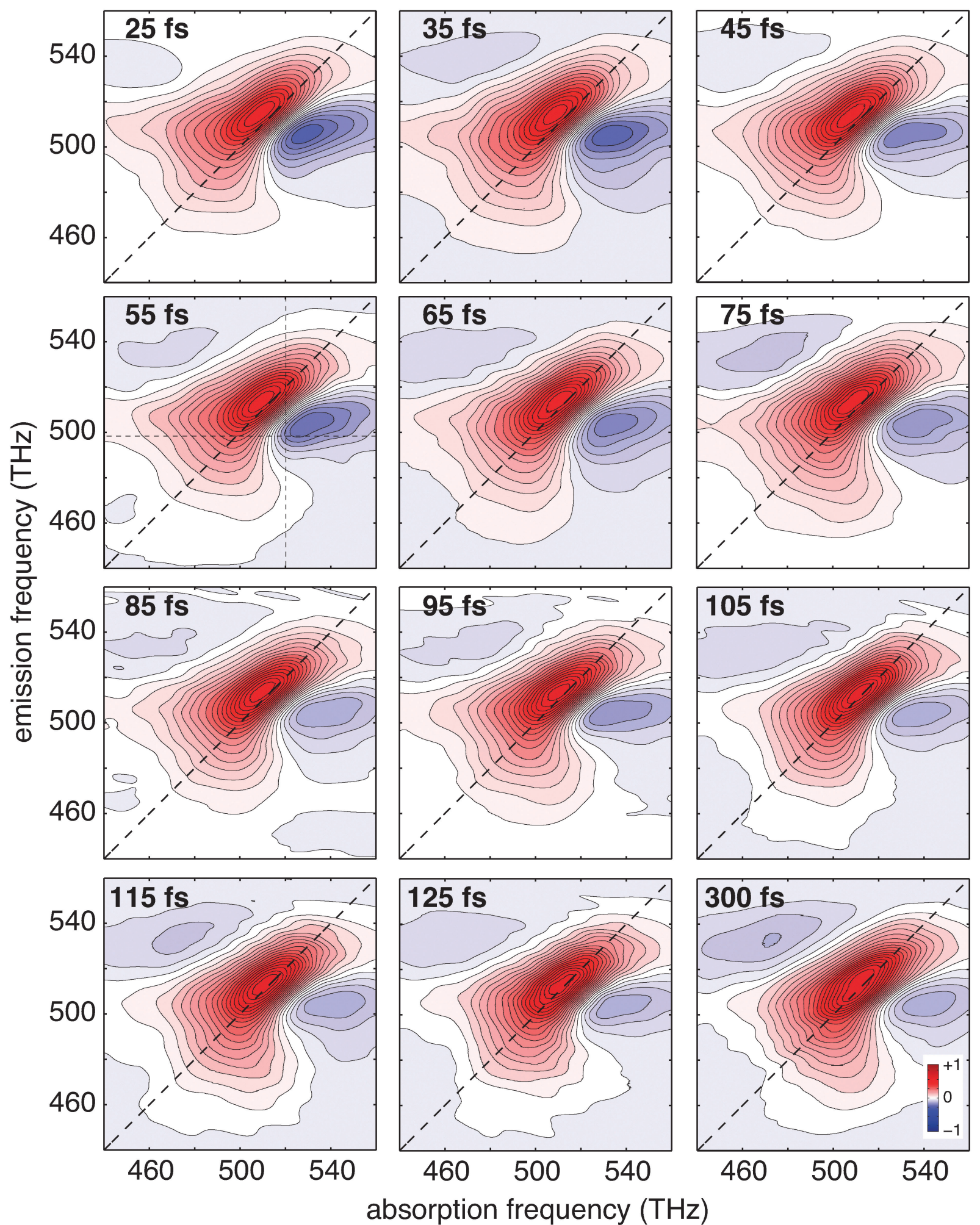

Fig. 8 The real part of the rephasing signal.

the line-outs presented in Fig. 7. Fifth, negative frequencies in $\nu_{2}$ are physically meaningful, and indicate the relative phase of the oscillation frequency. Sixth, as is apparent from the double-sided Feynman diagrams presented in Appendix 2 and discussed above, most but not all of the features will appear at locations in the $2 \mathrm{D}$ spectrum where $\nu_{3}-\nu_{1}=\nu_{2}$.
Selective integrations of the 3D spectral solid such as those presented in Fig. 6 are not commonly created or discussed. It is therefore appropriate to describe how to interpret such spectra. In short, the spectra show at which locations in the 2D spectrum certain frequencies are prominent. For example, the $-50 \mathrm{THz}$ projection shows the spectral locations where one 

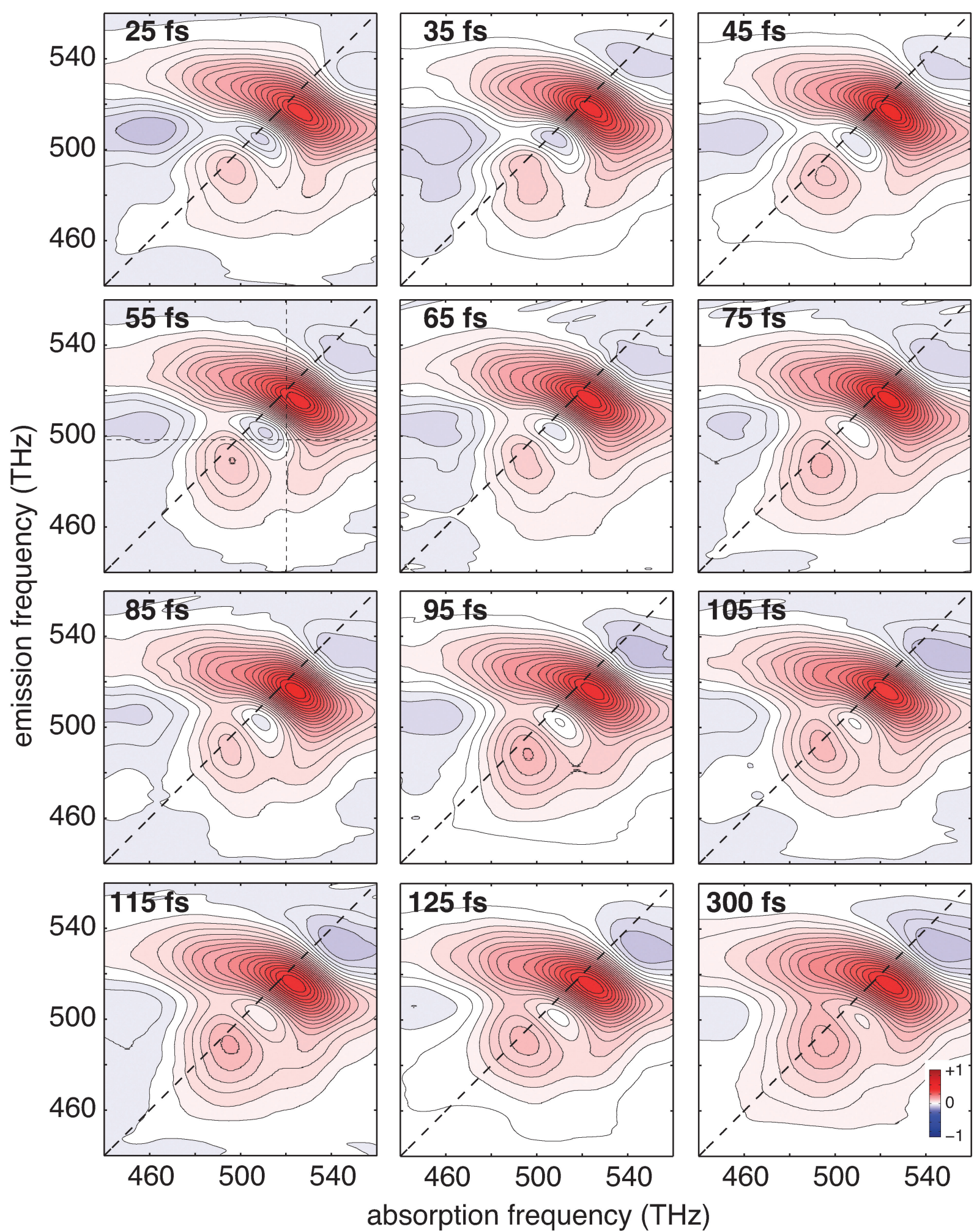

Fig. 9 The real part of the nonrephasing signal.

would find oscillations at this frequency. The cross peak is located at coordinates of $(525 \mathrm{THz}, 488 \mathrm{THz})$, and its shape may indicate that it is a split peak at two locations. It could be the same vibrational mode of two close-lying chromophores.

As another example, consider the $-40 \mathrm{THz}$ projection presented in Fig. 6, where the cross peak is located at (523 THz, $492 \mathrm{THz})$.
Again the peak shape is not round, and therefore is suggestive as being due to nearly overlapping signals from two close-lying chromophores. On the other hand, the cross peak in the $-26 \mathrm{THz}$ projection located at $(520 \mathrm{THz}, 500 \mathrm{THz})$ is round and appears to be slightly diagonally elongated. The peak shifts to coordinates of $(523 \mathrm{THz}, 499 \mathrm{THz})$ in the $-21 \mathrm{THz}$ projection, 
and the negative-amplitude signal due to ESA pathways is beginning to emerge from underneath the positive-amplitude features. The low-frequency projections show significantly greater ESA signals, and by $-7 \mathrm{THz}$, the cross-peak location is dominated by the ESA signal centred at a location of $(519 \mathrm{THz}$, $508 \mathrm{THz}$. The ESA peak highlighted in the $-13 \mathrm{THz}$ projection, displayed in Fig. 6, behaves much differently. It is elongated along the absorption dimension because the bandwidth truncates the features at that frequency. Thus, its lineshape in this measurement depended critically on the pulse spectrum.

The $\nu_{2}$ projections presented in Fig. 6 are striking in that the features are not spread about the spectrum but are instead prominent only near the cross-peak location. Even the $0 \mathrm{THz}$ and positive- $\nu_{2}$ projections show essentially no features above the diagonal where the emission frequency is greater than absorption frequency. For reference, other positivefrequency projections (which are not shown) are almost identical to the $+21 \mathrm{THz}$ projection. The asymmetric nature of the 2D spectrum of PC645 is probably meaningful and worthy of additional study. In model systems such as GaAs quantum wells, ${ }^{87} \mathrm{CdSe}$ quantum dots,${ }^{71}$ or laser dyes, ${ }^{10,42}$ the spectra are essentially symmetric about the diagonal. Based on measurement of asymmetric spectra of molecular nanotubes ${ }^{88-90}$ and other biological pigment-protein complexes, ${ }^{79,91-93}$ we speculate that an asymmetric 2D ES may be a signature of a system which involves both electronic and vibrational couplings.

\section{Conclusions}

Many questions surround the coherences observed in 2D ES studies of photosynthetic light-harvesting proteins. Here we used quantitative analysis of such measurements to answer some of those questions. In particular, our measurements showed that the $\mathrm{pH}$ drop in the lumen, which directly affects the environment of the light-harvesting proteins in cryptophyte algae, does not affect the observed coherent dynamics for the first $400 \mathrm{fs}$. We also found that under our excitation conditions, the cross-peak oscillation involves no fewer than eight different frequencies. Based on two discriminations between vibrational and electronic coherences, our analysis of the two clearest modes showed that the $21 \mathrm{THz}$ coherence, which had a dephasing time of $150 \pm 50 \mathrm{fs}$ at room temperature, was a signature of electronic coherence while the $26 \mathrm{THz}$ coherence was a signature of an intramolecular vibration. The six other modes were all of low amplitude relative to noise levels and thus could not be assigned using the differentiation protocols. We have also highlighted several areas - including the asymmetric nature of the spectra - where additional measurements and modelling are needed to understand the observed quantum coherence better.

\section{Appendix 1}

The first four figures of this appendix (Fig. 8 to 11) contain the real and imaginary parts of the separated rephasing and nonrephasing contributions to the total 2D ES data set, the real part of which is presented in Fig. 3. The representative 2D spectra are from one data set at $\mathrm{pH} 6.5$; thin dashed
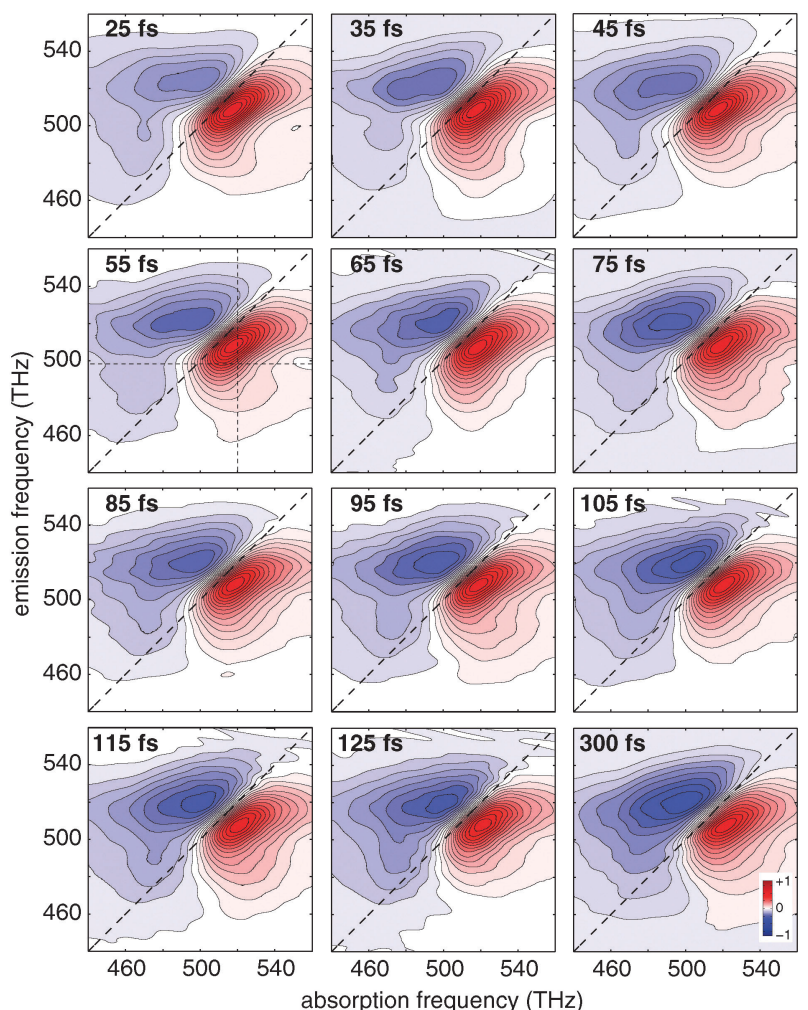

Fig. 10 The imaginary part of the rephasing signal.
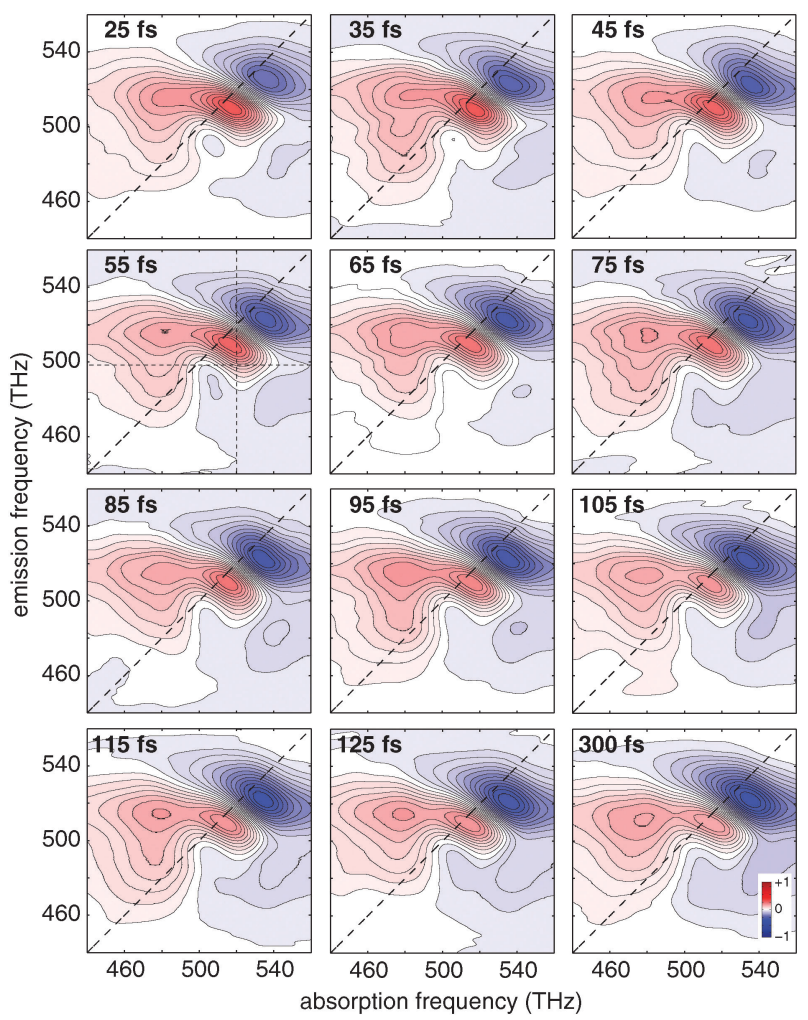

Fig. 11 The imaginary part of the nonrephasing signal.

vertical and horizontal lines in the $\tau_{2}=55 \mathrm{fs}$ spectra indicate the cross-peak extraction coordinates; and the spectra are individually normalized with 21 evenly spaced contours. 


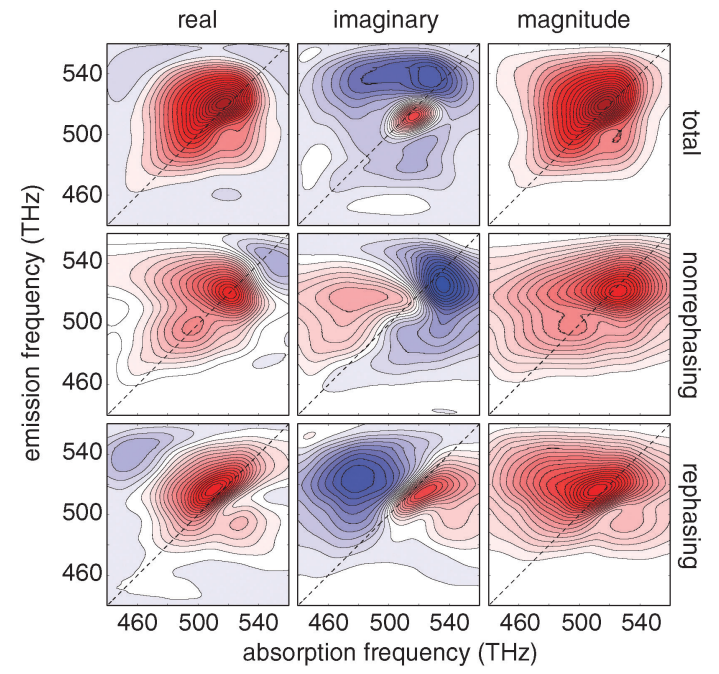

Fig. 12 The total 2D ES and its separated rephasing and nonrephasing components at $\tau_{2}=0 \mathrm{fs}$.
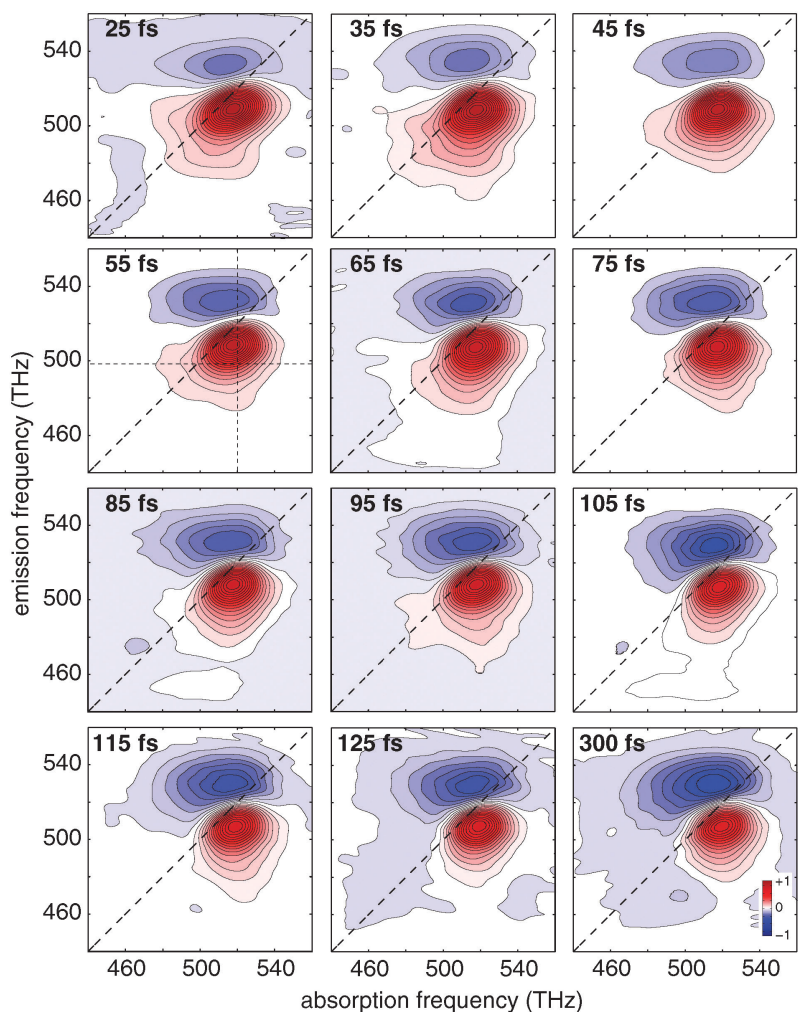

Fig. 13 The total imaginary 2D ES at the indicated $\tau_{2}$ time values.

In the fifth figure, Fig. 12, we display the total 2D ES and its separated rephasing and nonrephasing components measured at $\tau_{2}=0 \mathrm{fs}$. At this time, significant nonresonant response due to sample windows and solvent contributes to the measured signal.

The sixth figure, Fig. 13, displays the total imaginary 2D ES, similar to the total real 2D ES in Fig. 3.

\section{Appendix 2}

In Fig. 14(a)-(c), we present the energy-level scheme, a schematic spectrum for the case of two vibrational levels in ground and excited electronic states, and the set of doublesided Feynman diagrams, respectively. The diagrams in Fig. 14(c) are colour coded based on their location in the 2D spectrum in part (b): black diagrams indicate diagonal peaks while coloured diagrams indicate cross peaks. (The colour coding scheme is not related to the scheme used in Fig. 7.) Red underlines indicate diagrams that involve state $\left|g_{1}\right\rangle$. These diagrams, which account for half of the nonESA pathway diagrams, do not exist in our energy-level scheme used to describe electronic coherence (the three-level ' $\mathrm{V}$ '-shaped scheme). We find three features that are present in the four-level system (describing vibrational coherence) but not present in the three-level system (describing electronic coherence).

1. The orange and violet diagrams lead to signals which involve emission at an energy one vibrational quanta below the fundamental electronic absorption energy, $E_{3}=$ $e-\nu$. Producing such signals requires pulses with sufficient spectral brightness on the low-energy side of the main absorption peak.

2. The green cross peak involves oscillations at both $+\nu$ and $-\nu$, which will lead to a phase shift of this peak. Thus in the Fourier domain we would expect peaks at both positive and negative $\nu_{2}$ values for the green peak.

3. Both the green and blue cross peaks contain oscillations in the nonrephasing contribution. In addition, the rephasing contributions of the diagonal peaks will contain oscillations. However, because experiments typically contain a non-zero amount of unwanted scattered light, which appears on the diagonal, we prefer the test of oscillations in the nonrephasing contribution.

These three features are indications of vibrational coherence. If transitions to the $\left|\mathrm{g}_{1}\right\rangle$ state are not allowed — which would be the case in purely electronic coupling - these three features will not be present. Thus there are at least three differences between the signatures of pure electronic coherences and pure vibrational coherences. The excited-state absorption features are not depicted in the spectrum or in the Hamiltonian below.

The total Hamiltonian for this system, which is the sum of the material portion $\left(H_{\mathrm{m}}\right)$ and the transition-dipole-moment portion $\left(H_{\mathrm{f}}\right)$, can be written in the matrix form as

$$
H_{\mathrm{m}}+H_{\mathrm{f}}=\left[\begin{array}{cccc}
g_{0} & 0 & 0 & 0 \\
0 & g_{1} & 0 & 0 \\
0 & 0 & e_{0} & 0 \\
0 & 0 & 0 & e_{1}
\end{array}\right]+\left[\begin{array}{cccc}
0 & 0 & \mu_{0} & \mu_{+1} \\
0 & 0 & \mu_{-1} & \mu_{0} \\
\mu_{0} & \mu_{-1} & 0 & 0 \\
\mu_{+1} & \mu_{0} & 0 & 0
\end{array}\right]
$$

where the transition-dipole-moment matrix can be applied in a perturbative fashion after accounting for the electric field following the procedure outlined in many references, ref. 24 for example. In this treatment, we consider the vibrational quanta in the ground and excited states to be equal, $e_{1}-e_{0}=$ $g_{1}-g_{0}$. This treatment could easily be expanded to a more general form to account for differences in the ground and excited vibrational quanta.

We also note that some vibrational systems may not have allowed transitions to $\left|g_{1}\right\rangle$. The differentiation protocols rely on transitions involving this state. Thus, vibrational systems 
(a)

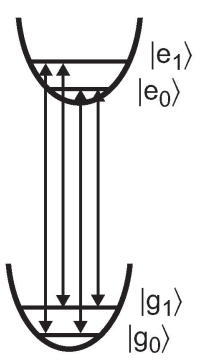

(b)

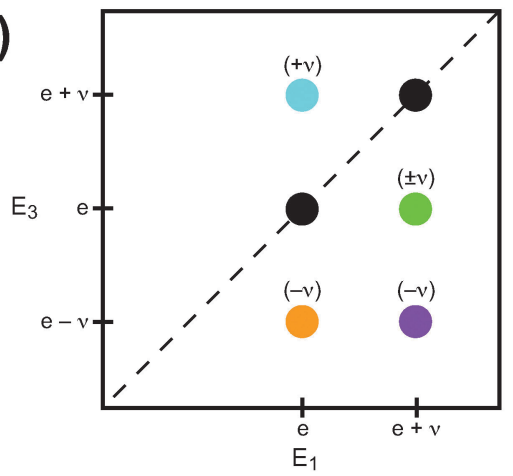

(c)
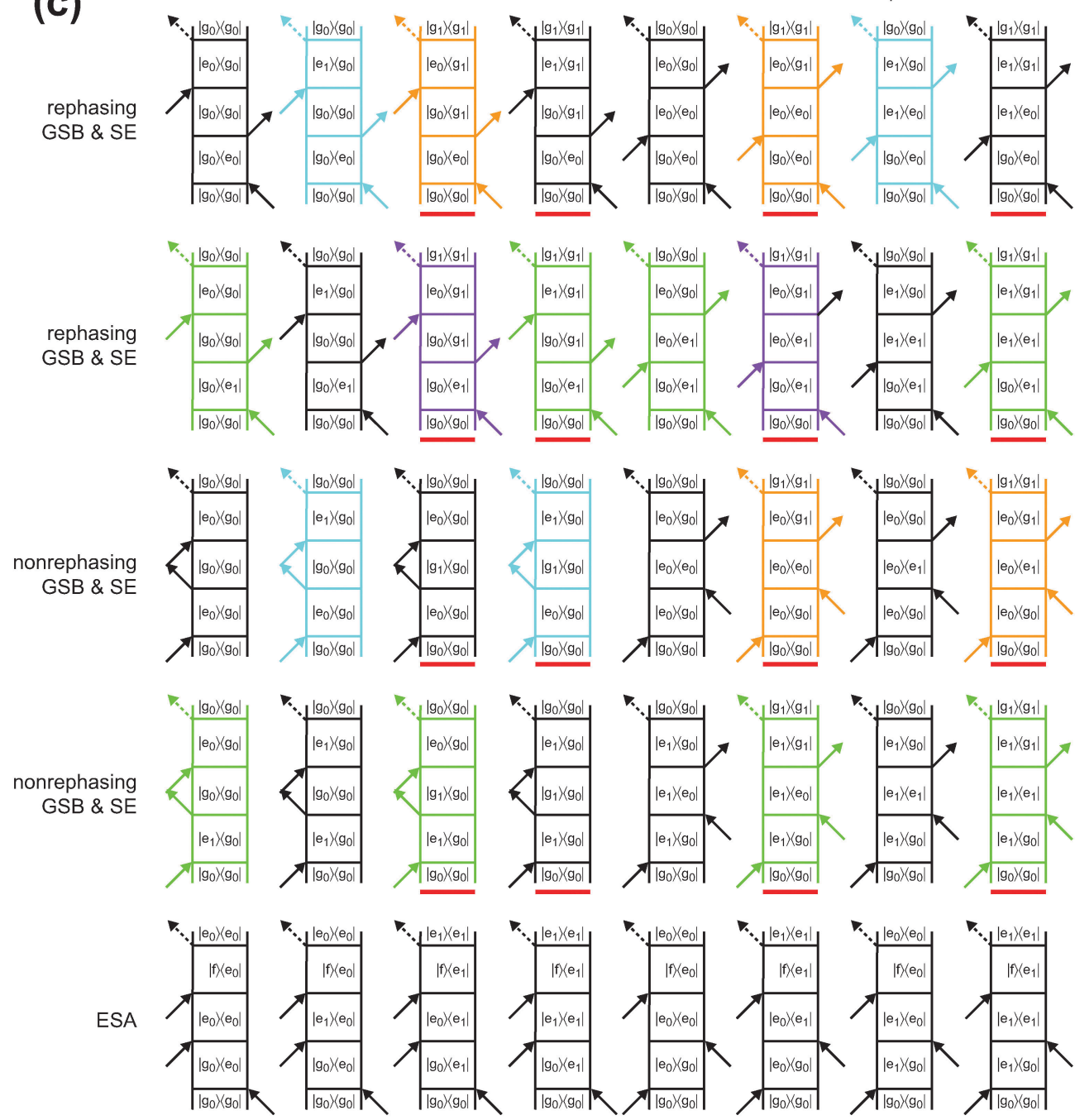

Fig. 14 Vibrational coherences. (a) Two vibrational levels $(|0\rangle$ and $|1\rangle)$ are indicated for each electronic state $(|\mathrm{g}\rangle$ and $|\mathrm{e}\rangle)$. There are four possible transitions among the four states. (b) The coordinates of the six predicted peaks. (c) The set of double-sided Feynman diagrams. Red underlines indicate diagrams that involve state $\left|\mathrm{g}_{1}\right\rangle$.

without transitions to $\left|\mathrm{g}_{1}\right\rangle$ would have 2D spectra identical to the model system for electronic coupling.

\section{Acknowledgements}

We acknowledge financial support from DARPA under the QuBE program, the United States Air Force Office of Scientific Research under contract number FA9550-10-1-0260, and the Natural Sciences and Engineering Research Council of Canada.
We thank Min-Duk Seo and Mitsuhiko Ikura for assistance with the CD measurements and Philip Johnson, Alexei Halpin, and Jürgen Hauer for helpful conversations regarding the 2D ES apparatus.

\section{References}

1 R. E. Blankenship, Molecular Mechanisms of Photosynthesis, Blackwell Scientific Publications, 2002.

2 G. D. Scholes, G. R. Fleming, A. Olaya-Castro and R. van Grondelle, Nat. Chem., 2011, 3, 763-774. 
3 M. Chachisvilis, O. Kühn, T. Pullerits and V. Sundström, J. Phys. Chem. B, 1997, 101, 7275-7283.

4 J. A. Cina and G. R. Fleming, J. Phys. Chem. A, 2004, 108, 11196-11208.

5 G. S. Engel, T. R. Calhoun, E. L. Read, T.-K. Ahn, T. Mancal, Y.-C. Cheng, R. E. Blankenship and G. R. Fleming, Nature, 2007, 446, 782-786.

6 I. P. Mercer, Y. C. El-Taha, N. Kajumba, J. P. Marangos, J. W. G. Tisch, M. Gabrielsen, R. J. Cogdell, E. Springate and E. Turcu, Phys. Rev. Lett., 2009, 102, 057402.

7 Y.-C. Cheng and G. R. Fleming, Annu. Rev. Phys. Chem., 2009, 60, 241-262.

8 E. Collini, C. Y. Wong, K. E. Wilk, P. M. G. Curmi, P. Brumer and G. D. Scholes, Nature, 2010, 463, 644-647.

9 G. Panitchayangkoon, D. Hayes, K. A. Fransted, J. R. Caram, E. Harel, J. Wen, R. E. Blankenship and G. S. Engel, Proc. Natl. Acad. Sci. U. S. A., 2010, 107, 12766-12770.

10 D. B. Turner, K. E. Wilk, P. M. G. Curmi and G. D. Scholes, J. Phys. Chem. Lett., 2011, 2, 1904-1911.

11 G. H. Richards, K. E. Wilk, P. M. G. Curmi, H. M. Quiney and J. A. Davis, J. Phys. Chem. Lett., 2012, 3, 272-277.

12 A. Ishizaki and G. R. Fleming, Proc. Natl. Acad. Sci. U. S. A., 2009, 106, 17255-17260.

13 F. Fassioli, A. Nazir and A. Olaya-Castro, J. Phys. Chem. Lett., 2009, 1, 2139.

14 X. Chen and R. J. Silbey, J. Chem. Phys., 2010, 132, 205101.

15 G. D. Scholes, J. Phys. Chem. Lett., 2010, 1, $2-8$.

16 P. Huo and D. F. Coker, J. Phys. Chem. Lett., 2011, 2, 825-833.

17 A. Kolli, A. Nazir and A. Olaya-Castro, J. Chem. Phys., 2011, 135, 154112.

18 M. Sarovar, A. Ishizaki, G. R. Fleming and K. B. Whaley, Nat. Phys., 2010, 6, 463- 467.

19 A. Ishizaki, T. R. Calhoun, G. Schlau-Cohen and G. R. Fleming, Phys. Chem. Chem. Phys., 2010, 12, 7319-7337.

20 A. Ishizaki and G. R. Fleming, J. Phys. Chem. B, 2011, 115, 6227-6233.

21 M. Tiersch, S. Popescu and H. J. Briegel, arxiv, 2011, 1104.3883.

22 D. M. Jonas, Annu. Rev. Phys. Chem., 2003, 54, 425-463.

23 D. M. Jonas, Science, 2003, 300, 1515-1517.

24 S. Mukamel, Principles of Nonlinear Optical Spectroscopy, Oxford University Press, New York, 1995.

25 M. Cho, Chem. Rev., 2008, 108, 1331-1418.

26 J. D. Hybl, A. W. Albrecht, S. M. Gallagher Faeder and D. M. Jonas, Chem. Phys. Lett., 1998, 297, 307-313.

27 J. D. Hybl, A. Albrecht Ferro and D. M. Jonas, J. Chem. Phys., 2001, 115, 6606-6622.

28 P. Tian, D. Keusters, Y. Suzaki and W. S. Warren, Science, 2003, 300, 1553-1555.

29 A. D. Bristow, D. Karaiskaj, X. Dai, T. Zhang, C. Carlsson, K. R. Hagen, R. Jimenez and S. T. Cundiff, Rev. Sci. Instrum., 2009, 80, 073108 .

30 N. S. Ginsberg, Y.-C. Cheng and G. R. Fleming, Acc. Chem. Res., 2009, 42, 1352-1363.

31 A. Nemeth, J. Sperling, J. Hauer, H. F. Kauffmann and F. Milota, Opt. Lett., 2009, 34, 3301-3303.

32 V. I. Prokhorenko, A. Halpin and R. J. D. Miller, Opt. Express, 2009, 17, 9764-9779.

33 D. B. Turner, K. W. Stone, K. Gundogdu and K. A. Nelson, Rev. Sci. Instrum., 2011, 82, 081301.

34 E. Harel, A. F. Fidler and G. S. Engel, J. Phys. Chem. A, 2011, 115, 3787-3796.

35 V. Novoderezhkin, R. Monshouwer and R. van Grondelle, J. Phys. Chem. B, 2000, 104, 12056-12071.

36 D. Egorova, M. F. Gelin and W. Domcke, Chem. Phys., 2007, 341, $113-122$.

37 A. Nemeth, F. Milota, T. Mancal, V. Lukes, H. F. Kauffmann and J. Sperling, Chem. Phys. Lett., 2008, 459, 94-99.

38 D. Egorova, Chem. Phys., 2008, 347, 166.

39 A. Nemeth, F. Milota, T. Mancal, V. Lukes, J. Hauer, H. F. Kauffmann and J. Sperling, J. Chem. Phys., 2010, 132, 184514.

40 T. Mancal, A. Nemeth, F. Milota, V. Lukes, J. Hauer, H. F. Kauffmann and J. Sperling, J. Chem. Phys., 2010, 132, 184515.

41 N. Christensson, B. Dietzek, A. Yartsev and T. Pullerits, Vib. Spectrosc., 2010, 53, 2-5.
42 N. Christensson, F. Milota, J. Hauer, J. Sperling, O. Bixner, A. Nemeth and H. F. Kauffmann, J. Phys. Chem. B, 2011, 115, 5383-5391.

43 J. F. Kern, J. Messinger and J. Whitmarsh, Encyclopedia of Life Sciences, Wiley-Blackwell, 2010.

44 B. Demming-Adamas and W. W. Adams, New Phytol., 2006, 172, $11-21$.

45 M. P. Johnson and A. V. Ruban, J. Biol. Chem., 2011, 286, 19973-19981.

46 C. Ilioaia, M. P. Johnson, C. D. P. Duffy, A. Pascal, R. van Grondelle, B. Robert and A. V. Ruban, J. Biol. Chem., 2011, 286, 91-98.

47 C. Funk, M. Alami, T. Tibiletti and B. R. G. Green, Biochim. Biophys. Acta, 2011, 1807, 841-846.

48 N. E. Holt, G. R. Fleming and K. K. Niyogi, Biochemistry, 2004, 43, 8281-8289.

49 X.-p. Li, A. M. Gilmore, S. Caffarri, R. Bassi, T. Golan, D. Kramer and K. K. Niyogi, J. Biol. Chem., 2004, 279, 22866-22874.

50 P. Müller, X.-p. Li and K. K. Niyogi, Plant Physiol., 2001, 125, $1558-1566$.

51 G. D. Scholes, G. R. Fleming, A. Olaya-Castro and R. van Grondelle, Nat. Chem., 2011, 3, 763-774.

52 A. B. Doust, K. E. Wilk, P. M. G. Curmi and G. D. Scholes, J. Photochem. Photobiol., A, 2006, 184, 1-17.

53 C. D. van der Weij-De Wit, A. B. Doust, I. H. M. van Stokkum, J. P. Dekker, K. E. Wilk, P. M. G. Curmi, G. D. Scholes and R. van Grondelle, J. Phys. Chem. B, 2006, 100, 25066-25073.

54 M. K. Yetzbacher, N. Belabus, K. A. Kitney and D. M. Jonas, J. Chem. Phys., 2007, 126, 044511.

55 R. MacColl, S. Kapoor, D. R. Montellese, S. Kukadia and L. E. Eisele, Biochemistry, 1996, 35, 15436-15439.

56 R. L. Fork, C. H. Brito Cruz, P. C. Becker and C. V. Shank, Opt. Lett., 1987, 12, 483-485.

57 S. Akturk, X. Gu, M. Kimmel and R. Trebino, Opt. Express, 2006, 14, 10101-10108.

58 N. Christensson, Y. Avlasevich, A. Yartsev, K. Müllen, T. Pascher and T. Pullerits, J. Chem. Phys., 2010, 132, 174508.

59 U. Brackmann, Lambdachrome Laser Dyes, Lambda Physik, 1997.

60 L. Lepetit, G. Cheriaux and M. Joffre, J. Opt. Soc. Am. B, 1995, 12, 2467-2474.

61 D. B. Turner, K. W. Stone, K. Gundogdu and K. A. Nelson, J. Chem. Phys., 2009, 131, 144510.

62 L. Yang, T. Zhang, A. D. Bristow, S. T. Cundiff and S. Mukamel, J. Chem. Phys., 2008, 129, 234711.

63 R. MacColl, H. Malak, J. Cipollo, B. Label, G. Ricci, D. MacColl and L. E. Eisele, J. Biol. Chem., 1995, 270, 27555-27561.

64 L. Spear-Bernstein and K. R. Miller, J. Phycol., 1989, 25, 412-419.

65 M. Debreczeny, Z. Gombos and B. Szalontai, Eur. Biophys. J., 1992, 21, 193-198.

66 B. Szalontai, Z. Gombos and M. Lutz, Photochem. Photobiol., 1994, 59, 574-578.

67 B. Szalontai, Z. Gombos, V. Csizmadia, C. Bagyinka and M. Lutz, Biochemistry, 1994, 33, 11823-11832.

68 T. Mirkovic, A. B. Doust, J. Kim, K. E. Wilk, C. Curutchet, B. Mennucci, R. Cammi, P. M. G. Curmi and G. D. Scholes, Photochem. Photobiol. Sci., 2007, 6, 964-975.

69 D. Hayes, J. Wen, G. Panitchayangkoon, R. E. Blankenship and G. S. Engel, Faraday Discuss., 2011, 150, 459-469.

70 D. B. Turner and K. A. Nelson, 2010 (unpublished).

71 D. B. Turner, Y. Hassan and G. D. Scholes, Nano Lett., 2011, DOI: $10.1021 / \mathrm{nl} 2039502$.

72 B. Szalontai, Z. Gombos and V. Csizmadia, Biochem. Biophys. Res. Commun., 1985, 130, 358-363.

73 J. Sawatzki, R. Fischer, H. Scheer and F. Siebert, Proc. Natl. Acad. Sci. U. S. A., 1990, 87, 5903-5906.

74 C. Kneip, A. Parbel, H. Foerstendorf, H. Scheer, F. Seibert and P. Hildebrandt, J. Raman Spectrosc., 1998, 29, 939-944.

75 C. Kneip, P. Hildebrandt, K. Nemeth, F. Mark and K. Schaffner, Chem. Phys. Lett., 1999, 311, 479-484.

76 F. Andel, J. T. Murphy, J. A. Haas, M. T. McDowell, I. van der Hoe, J. Lugtenburg, J. C. Lagarias and R. A. Mathies, Biochemistry, 2000, 39, 2667-2676.

77 M. A. Mroginski, F. Mark, W. Thiel and P. Hildebrandt, Biophys. J., 2007, 94, 1885-1894. 
78 J. M. Zhang, Y. J. Shiu, M. Hayashi, K. K. Liang, C. H. Chang, V. Gulbinas, C. M. Yang, T.-S. Yang, H. Z. Wang, Y.-T. Chen and S. H. Lin, J. Phys. Chem. A, 2001, 105, 8788-8891.

79 J. M. Womick and A. M. Moran, J. Phys. Chem. B, 2009, 113, 15747-15759.

80 J. M. Womick and A. M. Moran, J. Phys. Chem. B, 2011, 115, $1347-1356$.

81 V. Novoderezhkin, A. B. Doust, C. Curutchet, G. D. Scholes and R. van Grondelle, Biophys. J., 2010, 99, 344-352.

82 L. A. Pachón and P. Brumer, J. Phys. Chem. Lett., 2011, 2, $2728-2732$.

83 J. S. Briggs and A. Herzenberg, Mol. Phys., 1971, 21, 865-879.

84 A. Eisfeld and J. S. Briggs, Chem. Phys., 2006, 324, 376-384.

85 F. C. Spano, Acc. Chem. Res., 2010, 43, 429-439.

86 J. A. Davis, C. R. Hall, L. V. Dao, K. A. Nugent, H. M. Quiney, H. H. Tan and C. Jagadish, J. Chem. Phys., 2011, 135, 044510.
87 A. D. Bristow, D. Karaiskaj, X. Dai, R. P. Mirin and S. T. Cundiff, Phys. Rev. B: Condens. Matter, 2009, 79, 161305(R).

88 F. Milota, J. Sperling, A. Nemeth, D. Abramavicius, S. Mukamel and H. F. Kauffmann, J. Chem. Phys., 2009, 131, 054510.

89 F. Milota, J. Sperling, A. Nemeth, T. Mancal and H. F. Kauffmann, Acc. Chem. Res., 2009, 42, 1364-1374.

90 A. Nemeth, F. Milota, J. Sperling, D. Abramavicius, S. Mukamel and H. F. Kauffmann, Chem. Phys. Lett., 2009, 469, 130-134.

91 T. Brixner, J. Stenger, H. M. Vaswani, M. Cho, R. E. Blankenship and G. R. Fleming, Nature, 2005, 434, 625-628.

92 T. R. Calhoun, N. S. Ginsberg, G. Schlau-Cohen, Y.-C. Cheng, M. Ballottari, R. Bassi and G. R. Fleming, J. Phys. Chem. B, 2009 , 113, 16291-16295.

93 N. S. Ginsberg, J. A. Davis, M. Ballottari, Y.-C. Cheng, R. Bassi and G. R. Fleming, Proc. Natl. Acad. Sci. U. S. A., 2011, 108, 3848-3853. 